\title{
Predator-prey-subsidy population dynamics on stepping-stone domains with dispersal delays
}

\author{
Ragna M. Eide, Andrew L. Krause, Nabil T. Fadai, and Robert A. Van Gorder* \\ Mathematical Institute, University of Oxford, Andrew Wiles Building, Radcliffe Observatory Quarter, Woodstock Road, Oxford, OX2 6GG, UK \\ *Robert.VanGorder@maths.ox.ac.uk
}

\begin{abstract}
We examine the role of the travel time of a predator along a spatial network on predator-prey population interactions, where the predator is able to partially or fully sustain itself on a resource subsidy. The impact of access to food resources on the stability and behaviour of the predator-prey-subsidy system is investigated, with a primary focus on how incorporating travel time changes the dynamics. The population interactions are modelled by a system of delay differential equations, where travel time is incorporated as discrete delay in the network diffusion term in order to model time taken to migrate between spatial regions. The model is motivated by the Arctic ecosystem, where the Arctic fox consumes both hunted lemming and scavenged seal carcass. The fox travels out on sea ice, in addition to quadrennially migrating over substantial distances. We model the spatial predator-prey-subsidy dynamics through a "stepping-stone" approach. We find that a temporal delay alone does not push species into extinction, but rather may stabilize or destabilize coexistence equilibria. We are able to show that delay can stabilize quasi-periodic or chaotic dynamics, and conclude that the incorporation of dispersal delay has a regularizing effect on dynamics, suggesting that dispersal delay can be proposed as a solution to the paradox of enrichment.
\end{abstract}

Keywords: predator-prey dynamics; allochthonous resource subsidy; population dynamics; non-equilibrium dynamics; network structure in ecology; stepping-stone model; dispersal delay

\section{Introduction}

Interactions between the Arctic fox (Alopex lagopus) and the Arctic lemming (Cricetidae family) have traditionally been considered as a predator-prey system (Angerbjorn et al., 1999). However, the Arctic fox also consumes certain birds, bird eggs, and seal (Phocidae family) carrion discarded by polar bears (Ursus martimus) on the sea ice during migration (Nevai and Van Gorder, 2012; Roth, 2002, 2003). While the existence of an allochthonous resource in a predator-prey type interaction may seem innocuous, empirical studies have shown that resource subsidies can disrupt otherwise stable population interactions (Darimont et al., 2008; Halaj and Wise, 2002; Henden et al., 2010). Numerous other ecosystems follow the same interaction patterns, such as: systems where river otters benefit from a resource subsidy (Ben-David et al., 2005), the wolf, deer, and salmon carcass system (Adams et al., 2010; Darimont et al., 2008), and populations of puma that benefit from mule deer carcass in addition to hunted prey (Bauer et al., 2005).

One popular predator-prey model is the Rosenzweig and MacArthur (1963) model, given by

$$
\begin{aligned}
& \frac{d x}{d t}=r x\left(1-\frac{x}{k}\right)-\theta \frac{x y}{x+h}, \\
& \frac{d y}{d t}=\epsilon \theta \frac{x y}{x+h}-\delta y .
\end{aligned}
$$

Here, the birth rate of the prey is given by a logistic model with carrying capacity $k$ and growth rate $r$. The consumption rate is given by a Holling Type II functional response $\theta x /(x+h)$ (Turchin, 2003), such that the prey consumption rate saturates as $x$ grows large. The maximum prey consumption rate is denoted by $\theta, h$ is the half-saturation rate, $\epsilon$ is the efficiency of predator consumption, and $\delta$ is the death rate of the predator. The system admits a stable equilibrium for a certain range of the carrying capacity $k$, which undergoes a supercritical Hopf bifurcation as $k$ increases beyond this range (Hofbauer and Sigmund, 1998; Kot, 2001) and a limit cycle about the now unstable fixed point comes into existence. As $k$ increases further, the amplitude of the limit cycle grows and the prey density $x$ may periodically reach vanishingly small values which cannot persist in nature (Kot, 2001). This non-intuitive phenomena is known as the "paradox of enrichment" (Gilpin and Rosenzweig, 1972; Kot, 2001; May, 1972; Rosenzweig et al., 1971; Turchin, 2003). These limit cycles found under the Rosenzweig-MacArthur population model result in the "atto-fox problem", where solutions may fluctuate violently and reach arbitrarily small values, yet recover (Arditi and Berryman, 1991; Jensen and Ginzburg, 2005).

The Rosenzweig-MacArthur model was extended to include a resource subsidy by Nevai and Van Gorder (2012), in what was named the predator-prey-subsidy (PPS) model. Letting $s(t)$ denote the density of the resource subsidy at time $t$, the evolution of the prey, subsidy, and predator populations (denoted $x, s, y$, respectively) is described by the following system of dif- 
ferential equations:

$$
\begin{gathered}
\frac{d x}{d t}=r x\left(1-\frac{x}{k}\right)-\theta\left(\frac{x}{x+s+h}\right) y, \\
\frac{d s}{d t}=i-\gamma s-\psi\left(\frac{s}{x+s+h}\right) y, \\
\frac{d y}{d t}=\left(\frac{\epsilon \theta x+\eta \psi s}{x+s+h}\right) y-\delta y,
\end{gathered}
$$

where $i$ is the subsidy input rate, $\gamma$ is the rate at which the subsidy decays or is otherwise removed, and $\psi$ and $\eta$ are the subsidy equivalent of $\theta$ and $\epsilon$. Note that $h$ is now the half-saturation rate of combined prey and subsidy consumption. All other parameters are defined as in the Rosenzweig-MacArthur model, and all parameters are non-negative.

For sufficiently small subsidy input rates $i$, the model permits a stable predator-free equilibrium for small prey carrying capacities $k$, a stable positive equilibrium for intermediate $k$, and limit cycles for large $k$. The introduction of a subsidy is stabilizing in the sense that as $i$ increases from zero, the limit cycles appearing in the Rosenzweig-MacArthur model experience "amplitude death" (Nevai and Van Gorder, 2012). Moreover, as $i$ increases above a certain threshold, a unique, stable prey-free equilibrium point appears. Hence, high rates of resource subsidy input drives the prey to extinction.

The inclusion of non-autonomous periodic forcing terms in a Lotka-Volterra model has been shown to produce chaotic dynamics for certain parameter values (Zhang et al., 2015a). The PPS model was later extended by Levy et al. (2016) to include seasonal effects by introducing various time-dependent parameters. This non-autonomous PPS model displayed non-linear dynamics not found in the original model, such as quasi-periodic and chaotic oscillations.

\subsection{Including Spatial Dimension}

The models introduced above ignore spatial effects and assume random mixing on an isolated patch. However, the Arctic fox is a migratory animal and migrates every three or four years, often taking random paths rather than migrating in known patterns (Wrigley and Hatch, 1976). This migration takes about five months, during which the fox suffers from higher death rates due to lack of food and other hazards (Wrigley and Hatch, 1976). Moreover, the fox must travel onto the sea ice to consume seal carrion, into an area where their natural prey, the lemming, does not live. Therefore, we consider the impact of predator movement on the population dynamics.

Models that investigate spatial and migratory effects can generally be categorized into one of three groups: island models, stepping-stone models, and continuum models (Shen and Van Gorder, 2017). An $n$-patch island model considers a set of $n$ spatially separated environments, where, on each patch, populations exist and are governed by a system of equations such as those described above. As there is no movement between patches, the relative locations of different populations have no effect on the model behavior (Kareiva et al., 1990; Levin, 1976). An $n$-patch stepping-stone model assigns a spatial coordinate to every patch and a network of permissible routes of movement. The migratory populations may move across the network by discrete diffusion. It is generally assumed that migration is instantaneous, meaning travel time is not incorporated in the model. Notice that the relative spatial location of each patch is now explicitly included in the model, and stepping-stone domains differ from island domains in that the spatial structure may influence the dynamics (Neubert et al., 2002; Shen and Van Gorder, 2017). Lastly, continuum models use partial differential equations to describe spatial dispersal of populations in a continuous domain (Bassett et al., 2017; Kareiva et al., 1990; Levin, 1976).

Substantial research has been done on the effect of dispersion across spatial domains of predator prey models (Jansen, 1995, 2001; Jansen et al., 2000; Levin, 1974, 1976; Scheffer and De Boer, 1995; Weisser and Hassell, 1996). It has been suggested that population dispersion, of either predator, prey, or both species, has a stabilizing effect on the dynamics of the model, causing amplitude death of limit cycles and dampening the effect of the paradox of enrichment (Jansen, 1995). While a system with two competing prey-populations and one predator in a single patch exhibit enriched dynamics and even chaotic oscillations for physical parameter regimes (Hutson and Vickers, 1983), a two-patch system with a separate prey-population on each patch with a migratory predator was found to be stabilized by dispersion of the predator (Holt, 1984).

However, modelling spatial dispersion increases the dimensionality of the model, which may give rise to more complex dynamics. In (Jansen, 2001), Jansen investigated a two-patch Rosenzweig-MacArthur model with a predator and prey population on each patch, where the predator was allowed to migrate by discrete diffusion. Quasi-periodic solutions and chaotic attractors were observed, where unphysically large carrying capacities $k$ were required for the latter behavior. For high rates of dispersal, solutions existed where the prey were driven to extinction in one patch only. More sophisticated models of dispersion that incorporate animal behaviors, such as predator aversion or predation, have also been investigated for a two-patch predator-prey model by making dispersion density-dependent (Hauzy et al., 2010; Kang et al., 2015).

Nevai and Van Gorder (2012) proposed a two-patch island model, where the prey was confined to one patch and the subsidy to another. The predator was allowed to travel instantaneously between the two domains by discrete diffusion at rate $\alpha$. The two-patch PPS model was also motivated by the Arctic ecosystem, where the prey is non-migratory while the predator both travels out on the ice in search of resource subsidy and migrates over longer distances quadrennially (Dalén et al., 2004; Roth, 2002, 2003). The effect of the subsidy on the system was similar to that of the single-patch case and it was found that increasing the dispersion rate $\alpha$ also had a stabilizing effect on the system (Nevai and Van Gorder, 2012).

A general $n$-patch PPS model was proposed by Levy et al. (2016). Letting $x^{(j)}(t), s^{(j)}(t), y^{(j)}(t)$ be the population densities 
at patch $\mathbb{P}^{(j)}$ at time $t$, the model is given by

$$
\begin{gathered}
\frac{d x^{(j)}}{d t}=r^{(j)} x^{(j)}\left(1-\frac{x^{(j)}}{k^{(j)}}\right)-\theta^{(j)}\left(\frac{x^{(j)}}{x^{(j)}+s^{(j)}+h^{(j)}}\right) y^{(j)}, \\
\frac{d s^{(j)}}{d t}=i^{(j)}-\gamma^{(j)} s^{(j)}-\psi^{(j)}\left(\frac{s^{(j)}}{x^{(j)}+s^{(j)}+h^{(j)}}\right) y^{(j)}, \\
\frac{d y^{(j)}}{d t}=\left(\frac{\epsilon^{(j)} \theta^{(j)} x^{(j)}+\eta^{(j)} \psi^{(j)} s^{(j)}}{x^{(j)}+s^{(j)}+h^{(j)}}\right) y^{(j)}-\delta^{(j)} y^{(j)} \\
+\sum_{\ell=1}^{n}\left(\lambda^{(\ell, j)} \alpha^{(\ell, j)} y^{(\ell)}-\alpha^{(j, \ell)} y^{(j)}\right)
\end{gathered}
$$

for $j \in\{1,2, \ldots n\}$. Here, $\alpha^{(\ell, j)}$ is the dispersion rate from patch $\mathbb{P}^{(\ell)}$ to $\mathbb{P}^{(j)}$ and $\lambda^{(\ell, j)}$ is the survival rate of the predator upon moving from $\mathbb{P}^{(\ell)}$ to $\mathbb{P}^{(j)}$. Migration from a patch to itself is disallowed, so $\alpha^{(j, j)}=0$ for all $j$. As the focus of Levy et al. (2016) was on the impact of non-autonomous parameters, only simple two- and three-node networks were considered. Shen and Van Gorder (2017) found that network structure had some influence on the dynamics of the $n$-patch PPS system. Increasing migration rates $\alpha^{(\ell, j)}$ was largely stabilizing, and could drive the predator to extinction in most network structures where access to food was scarce. However, it was also found that in some cases where predator death would occur in a single-patch model, having multiple patches could allow the predator to survive. Moreover, there exists a family of networks for which increasing $\alpha$ leads to destabilization akin to the paradox of enrichment (Shen and Van Gorder, 2017).

\subsection{Delayed Migration}

The models discussed so far assume that migration is instantaneous between patches. While this assumption may be appropriate in systems where the time-scale of migration is fast in comparison to the dynamics on the patches, this is not the case for our motivating example of the Arctic ecosystem. Moreover, we may want to differentiate migration of the fox between island regions to trips out on the ice, which have different duration. Hence, incorporating delayed migration into the model is a way of further describing spatial information. Although dispersion is a well-studied topic, inclusion of travel time as delay is less so (Zhang et al., 2015b). Wall et al. (2013) show that even small delays can have a large impact on the qualitative behavior of a biological system, implying that the assumption of instantaneous travel may be illegitimate. Introducing delay into dynamical systems is generally destabilizing (MacDonald and MacDonald, 2008), and delay in the inter-species interaction terms of a predator-prey model has been shown to cause chaotic behavior (Choudhury, 1992).

Delayed migration with one patch has been studied by removing the migrating predators from the predator-prey dynamics through a "dispersal pool" (Holt, 1984; Weisser and Hassell, 1996; Weisser et al., 1997). This can be considered as a two-patch system with discrete dispersal migration where one patch is barren. Similarly, delayed migration in a PPS model was investigated by Levy et al. (2016), where they considered a three-patch linearly linked system where $\mathbb{P}^{(1)}$ was subsidy-free,
$\mathbb{P}^{(2)}$ prey- and subsidy-free, and $\mathbb{P}^{(3)}$ prey-free. Both studies found that this type of delayed migration is always stabilizing (Holt, 1984; Levy et al., 2016). While using one or more barren patches to describe distance between habitats is an intuitive approach, it makes unnatural assumptions about the way migration occurs. In particular, it implies that there is an exponential distribution of travel times. Hence, there are no minimum or maximum trip duration and a predator could hypothetically survive an arbitrarily long trip (Neubert et al., 2002).

Predator-prey systems described by stepping-stone LotkaVolterra models with dispersal delay and reduced migration survival rate were investigated by Neubert et al. (2002). These modifications were stabilizing compared to standard stepping-stone Lotka-Volterra models for non-discrete travel times. Klepac et al. (2007) studied a single-patch RosenzweigMacArthur model, where the predator migrates with arbitrary distributions of trip durations. It was shown that for most distributions, time delay was stabilizing. However, for discrete travel times, quasi-periodic and aperiodic behavior was observed in addition to the existence of multiple attractors for certain values of the delay $\tau$. Specifically, a hysteresis effect was observed when the time delay $\tau$ was varied, where, depending on the initial conditions, both stable equilibria and aperiodic solutions could exist (Klepac et al., 2007). The two-patch RosenzweigMacArthur predator-prey model with delayed passive prey migration and homogeneous patches was investigated by Zhang et al. (2015b), where delay was found to always be stabilizing.

\subsection{Overview}

The predator species' access to food resources are determined by the migration rate, travel time, and rate of survival during migration. We aim to further the understanding of how changes to this access impacts the dynamics of the PPS model and hence more realistic predator-prey interactions in general. As was argued in Section 1.2, delayed migration better describes the relative distance between patches compared to instantaneous travel. We will consider the impact of delayed dispersion on the PPS system, focusing on the two-patch model of Nevai and Van Gorder (2012) for simplicity. The remainder of the paper is organized as follows. In Section 2, we formulate the multi-patch stepping-stone models with dispersal delays. In Section 3, we study the qualitative dynamics and bifurcations emergent from two-patch models, showing that dispersal delays can stabilize the dynamics for some parameter regimes, and then we extend these results to specific stepping-stone domains with more than two nodes in Section 4 to ascertain the role of the underlying spatial network structure on the emergent dynamics. In Section 5, we demonstrate how the inclusion of dispersal delays can regularize quasi-periodic and chaotic dynamics, in some cases resolving the paradox of enrichment by ensuring that populations are bounded far enough away from zero to prevent extinction. We discuss the results and their biological implications in Section 6. Conclusions and directions for future work are finally given in Section 7. 


\section{Multi-patch Stepping-Stone Models}

Consider a modification to the $n$-patch model presented by Levy et al. (2016) with migration delay incorporated. The $n$ patch model has populations of predator, prey, and subsidy species distributed over a network of $n$ nodes. Each node or patch is defined as a closed region of space where the local population dynamics are not directly influenced by the behavior in other patches. The prey and subsidy are confined to the patch where they originate from, while the predators may move across the network.

Denote the patches by $\mathbb{P}^{(j)}$ for $j \in\{1, \ldots, n\}$. Let $x^{(j)}(t), s^{(j)}(t)$, and $y^{(j)}(t)$ be the populations of prey, subsidy, and predators in $\mathbb{P}^{(j)}$ at time $t$, respectively. The populations are modelled by the following system of equations for each $j$ :

$$
\begin{aligned}
\frac{d x^{(j)}}{d t}= & r^{(j)} x^{(j)}\left(1-\frac{x^{(j)}}{k^{(j)}}\right)-\theta^{(j)}\left(\frac{x^{(j)}}{x^{(j)}+s^{(j)}+h^{(j)}}\right) y^{(j)}, \\
\frac{d s^{(j)}}{d t}= & i^{(j)}-\gamma^{(j)} s^{(j)}-\psi^{(j)}\left(\frac{s^{(j)}}{x^{(j)}+s^{(j)}+h^{(j)}}\right) y^{(j)}, \\
\frac{d y^{(j)}}{d t}= & \left(\frac{\epsilon^{(j)} \theta^{(j)} x^{(j)}+\eta^{(j)} \psi^{(j)} s^{(j)}}{x^{(j)}+s^{(j)}+h^{(j)}}\right) y^{(j)}-\delta^{(j)} y^{(j)} \\
& +\sum_{\ell=1}^{n}\left(\lambda^{(\ell, j)} \alpha^{(\ell, j)} y^{(\ell)}\left(t-\tau^{(\ell, j)}\right)-\alpha^{(j, \ell)} y^{(j)}\right) .
\end{aligned}
$$

Here, the time dependence of $x^{(j)}, s^{(j)}$, and $y^{(j)}$ is suppressed except for in terms with delay. The parameters are analogous to those of the PPS $n$-patch model. We have added the parameter $\tau^{(\ell, j)}$, the travel time from $\mathbb{P}^{(\ell)}$ to $\mathbb{P}^{(j)}$. We require $\tau^{(\ell, j)} \geq 0$ for all $\ell$ and $j$. Note that $\tau^{(\ell, j)}$ may not be equal to $\tau^{(j, \ell)}$. All parameters are non-negative, $\epsilon^{(j)}, \eta^{(j)}$, and $\lambda^{(\ell, j)}$ are bounded above by unity for biological reasons, and we make the assumption that $h^{(j)}>0$. Although $h^{(j)}, \theta^{(j)}, \psi^{(j)}, \epsilon^{(j)}$, and $\eta^{(j)}$ may vary between patches, we will consider them fixed across the system for the remainder of this paper, so we drop the $(j)$-dependence hereafter.

\subsection{Nondimensionalization}

Assume we have the $n$-patch model presented in Equations (9)-(11) with $r^{(j)}=r$ for all $j$, meaning the birth rate of the prey is the same across all patches. Define $\hat{t}=r t, x^{(j)}=h \hat{x}^{(j)}, s^{(j)}=$ $h \hat{S}^{(j)}$, and $y^{(j)}=v \hat{y}^{(j)}$, where $v=h r / \theta$. Let $\hat{i}^{(j)}=i^{(j)} /(h r), \hat{\gamma}^{(j)}=$ $\gamma^{(j)} / r, \hat{\delta}^{(j)}=\delta^{(j)} / r, \hat{\alpha}^{(\ell, j)}=\alpha^{(\ell, j)} / r$, be the supply and decay rate of subsidy, and death rate and dispersion rate of the predator in the new timescale. The rescaled travel time is $\hat{\tau}^{(j)}=\tau^{(j)} / r$, and $\hat{\epsilon}=\epsilon \theta / r, \hat{\eta}=\eta \psi / r$. Due to our choices of homogeneous parameters between patches, we define the nondimensionalized migration survival rate as $\hat{\lambda}^{(\ell, j)}=\lambda^{(\ell, j)} v^{(\ell)} / v^{(j)}=\lambda^{(\ell, j)}$. If $h, r$, and $\theta$ vary across patches, $\hat{\lambda}^{(\ell, j)}$ is a measure of the survival rate during migration that incorporates the differences between patches. Making these substitutions and dropping the hats on the variables for ease of reading, Equations (9)-(11) become

$$
\frac{d x^{(j)}}{d t}=x^{(j)}\left(1-\frac{x^{(j)}}{\kappa^{(j)}}\right)-\left(\frac{x^{(j)}}{x^{(j)}+s^{(j)}+1}\right) y^{(j)},
$$

$$
\begin{aligned}
\frac{d s^{(j)}}{d t}= & i^{(j)}-\gamma^{(j)} s^{(j)}-\phi\left(\frac{s^{(j)}}{x^{(j)}(t)+s^{(j)}+1}\right) y^{(j)}, \\
\frac{d y^{(j)}}{d t}= & \left(\frac{\epsilon x^{(j)}+\eta s^{(j)}}{x^{(j)}+s^{(j)}+1}\right) y^{(j)}-\delta^{(j)} y^{(j)} \\
& +\sum_{\ell=1}^{n}\left(\lambda^{(\ell, j)} \alpha^{(\ell, j)} y^{(\ell)}\left(t-\tau^{(\ell, j)}\right)-\alpha^{(j, \ell)} y^{(j)}\right),
\end{aligned}
$$

where $\kappa^{(j)}=k^{(j)} / h$ is a scaled carrying capacity and $\phi=\psi / \theta$ is a measure of the relative consumption rates of subsidy and prey for the predator. The inclusion of this scaling factor $v^{(\ell)} / v^{(j)}$ into $\lambda^{(\ell, j)}$ allows us to choose different expressions for $v$ at different patches. Note that while the re-scaling of $x, s$, and $y$ may be different at every patch, it is essential that $r^{(j)}=r$ for all patches, or else the timescale would not be consistent throughout the domain.

\subsection{Separated n-Patch Model}

We consider a variation on the general $n$-patch model, where the set of patches $\left\{\mathbb{P}^{(j)}\right\}_{j=1}^{n}$ is the union of three non-overlapping subsets $\mathcal{N}_{S}, \mathcal{N}_{X}$, and $\mathcal{N}_{E} . \mathcal{N}_{S}$ and $\mathcal{N}_{X}$ will be sets of patches only populations of subsidy and prey permanently inhabit, respectively, and $\mathcal{N}_{E}$ will be the set of patches without any food source for the predator. The predator is still allowed to move over all patches $\mathbb{P}^{(j)}$. In the language of our model, $x=0$ on patches in $\mathcal{N}_{S}, s=0$ on patches in $\mathcal{N}_{X}$, and $x=s=0$ in $\mathcal{N}_{E}$ for all time $t \geq 0$. The inspiration for this variation of the main model comes from the origins of the Predator-Prey-Subsidy Model; as the arctic foxes would travel from their habitat onto the ice to consume seal carcasses, they travel from patches with only prey (lemmings) to ones with only resource subsidy (seal).

We only give the nondimensional model here. We have for $\mathbb{P}^{(j)} \in \mathcal{N}_{X}$,

$$
\begin{aligned}
\frac{d x^{(j)}}{d t}= & x^{(j)}\left(1-\frac{x^{(j)}}{\kappa^{(j)}}\right)-\left(\frac{x^{(j)}}{x^{(j)}+1}\right) y^{(j)}, \\
\frac{d y^{(j)}}{d t}= & \left(\frac{\epsilon x^{(j)}}{x^{(j)}+1}\right) y^{(j)}-\delta^{(j)} y^{(j)} \\
& +\sum_{\ell=1}^{n}\left(\lambda^{(\ell, j)} \alpha^{(\ell, j)} y^{(\ell)}\left(t-\tau^{(\ell, j)}\right)-\alpha^{(j, \ell)} y^{(j)}\right),
\end{aligned}
$$

for $\mathbb{P}^{(j)} \in \mathcal{N}_{S}$,

$$
\begin{aligned}
\frac{d s^{(j)}}{d t}= & i^{(j)}-\gamma^{(j)} s^{(j)}-\phi\left(\frac{s^{(j)}}{s^{(j)}+1}\right) y^{(j)}, \\
\frac{d y^{(j)}}{d t}= & \left(\frac{\eta s^{(j)}}{s^{(j)}+1}\right) y^{(j)}-\delta^{(j)} y^{(j)} \\
& +\sum_{\ell=1}^{n}\left(\lambda^{(\ell, j)} \alpha^{(\ell, j)} y^{(\ell)}\left(t-\tau^{(\ell, j)}\right)-\alpha^{(j, \ell)} y^{(j)}\right),
\end{aligned}
$$

and for $\mathbb{P}^{(j)} \in \mathcal{N}_{E}$,

$$
\frac{d y^{(j)}}{d t}=-\delta^{(j)} y^{(j)}+\sum_{\ell=1}^{n}\left(\lambda^{(\ell, j)} \alpha^{(\ell, j)} y^{(\ell)}\left(t-\tau^{(\ell, j)}\right)-\alpha^{(j, \ell)} y^{(j)}\right) .
$$


We give technical details on the existence and boundedness of solutions to (15)-(19) in Appendix A to demonstrate that the model gives biologically reasonable solutions.

We note that (15)-(19) has different stability properties to (12)-(14) and hence should be considered independently. To demonstrate this, we shall contrast the separated and general two-patch models without delay $(\tau=0)$. The separated two-patch model was analyzed in great detail in Nevai and Van Gorder (2012). We will refer to this analysis and its results here. For the general model, both $\mathbb{P}^{(1)}$ and $\mathbb{P}^{(2)}$ may have populations of predator, prey, or subsidy species. Let $i^{(1)}=0, i^{(2)}>0$, and all other parameter values be constant and identical across the patches, so that $\mathbb{P}^{(1)}$ is subsidy-free. The model is defined by Equations (12)-(14) for $n=2$. Hence, it is a system of six coupled, nonlinear differential equations.

Let us order the state variables as $\left(x^{(1)}, s^{(1)}, y^{(1)}, y^{(2)}, s^{(2)}, x^{(2)}\right)$, which will allow for a convenient expression of the Jacobian. Let $D_{1}, D_{2}$ denote $x_{*}^{(1)}+s_{*}^{(1)}+1$ and $x_{*}^{(2)}+s_{*}^{(2)}+1$, respectively. At a fixed point $\left(x_{*}^{(1)}, s_{*}^{(1)}, y_{*}^{(1)}, y_{*}^{(2)}, s_{*}^{(2)}, x_{*}^{(2)}\right)$, we have the Jacobian

$$
J=\left[\begin{array}{ll}
J_{1} & J_{2} \\
J_{2}^{\top} & J_{3}
\end{array}\right],
$$

where the submatrices are given by

$J_{1}=\left[\begin{array}{ccc}1-\frac{2 x_{*}^{(1)}}{\kappa}-\frac{\left(s_{*}^{(1)}+1\right) y_{*}^{(1)}}{D_{1}^{2}} & \frac{x_{*}^{(1)} y_{*}^{(1)}}{D_{1}^{2}} & -\frac{x_{*}^{(1)}}{D_{1}} \\ -\phi \frac{s_{*}^{(1)} y_{*}^{(1)}}{D_{1}^{2}} & -\gamma-\phi \frac{\left(x_{*}^{(1)}+1\right) y_{*}^{(1)}}{D_{1}^{2}} & -\phi \frac{s_{*}^{(1)}}{D_{1}} \\ \frac{\left((\epsilon-\eta) s_{*}^{(1)}+\epsilon\right) y_{*}^{(1)}}{D_{1}^{2}} & \frac{\left((\eta-\epsilon) x_{*}^{(1)}+\eta\right) y_{*}^{(1)}}{D_{1}^{2}} & \frac{\epsilon x_{*}^{(1)}+\eta s_{*}^{(1)}}{D_{1}}-\delta-\alpha\end{array}\right]$,

$J_{2}=\left[\begin{array}{ccc}0 & 0 & 0 \\ 0 & 0 & 0 \\ \lambda \alpha & 0 & 0\end{array}\right]$,

$J_{3}=\left[\begin{array}{ccc}\frac{\epsilon x_{*}^{(1)}+\eta s_{*}^{(1)}}{D_{2}}-\delta-\alpha & \frac{\left((\eta-\epsilon) x_{*}^{(1)}+\eta\right) y_{*}^{(1)}}{D_{2}^{2}} & \frac{\left((\epsilon-\eta) s_{*}^{(1)}+\epsilon\right) y_{*}^{(1)}}{D_{2}^{2}} \\ -\phi \frac{s_{*}^{(2)}}{D_{2}} & -\gamma-\phi \frac{\left(x_{*}^{(2)}+1\right) y_{*}^{(2)}}{D_{2}^{2}} & -\phi \frac{s_{*}^{(2)} y_{*}^{(2)}}{D_{2}^{2}} \\ -\frac{x_{*}^{(2)}}{D_{2}} & \frac{x_{*}^{(2)} y_{*}^{(2)}}{D_{2}^{2}} & 1-\frac{2 x_{*}^{(2)}}{\kappa}-\frac{\left(s_{*}^{(2)}+1\right) y_{*}^{(2)}}{D_{2}^{2}}\end{array}\right]$.

In the separated two-patch model without delay, the predatorfree fixed point is stable when the parameters satisfy certain constraints (Nevai and Van Gorder, 2012). The fixed point is $x_{*}=\kappa$ and $s_{*}=i / \gamma$. In that case, one patch has only subsidy and the other has only prey residing on it. In order to replicate this behavior in the general two-patch model, we consider the case where $\mathbb{P}^{(1)}$ is prey-only, so that $x^{(1)}=\kappa, s^{(1)}=0$, and $i^{(1)}=0$, and $\mathbb{P}^{(2)}$ is subsidy only, meaning $x^{(2)}=0$ and $s^{(2)}=i / \gamma$. The Jacobian is now given by

$$
J=\left[\begin{array}{ccccc|c}
-1 & 0 & * & 0 & 0 & 0 \\
0 & * & 0 & 0 & 0 & 0 \\
0 & 0 & * & * & 0 & 0 \\
0 & 0 & * & * & 0 & 0 \\
0 & 0 & 0 & * & * & 0 \\
\hline 0 & 0 & 0 & 0 & 0 & 1
\end{array}\right]
$$

As $\mu=1$ is an eigenvalue of the Jacobian, this is an unstable fixed point for all parameter values. This is because any introduction of prey to $\mathbb{P}^{(2)}$ will induce growth in the prey population. Due to our nondimensionalization, we are unable to set the reproduction rate to zero and we cannot set the carrying capacity to zero. Hence, the only way to ensure that prey may not inhabit $\mathbb{P}^{(2)}$ is to set $x^{(2)}(0)=0$ and $d x^{(2)} / d t=0$.

It follows that the separated two-patch model has different stability-properties than the general two-patch model.

\section{Numerical Simulations of the Two-Patch Model}

We present results obtained numerically for the two-patch separated PPS model. These include both simulations of the system of delay-differential equations, and numerical stability analysis of the two-patch system. In general, accounting for environmental heterogeneity is important to understand ecosystem dynamics (Goldwyn and Hastings, 2009; Yang et al., 2015). However, as a first attempt to understand the interactions between each component of our model, we take homogeneous parameters across the patches, and therefore drop the superscripts. We note that even with this simplification, there are many remaining nondimensional parameters in the model, which can vary widely depending on the ecosystem being modelled. These parameters can be estimated either a priori based on biological considerations, such as allometric scaling, or $a$ posteriori based on observed populations over time; these approaches have been compared using a delayed logistic growth model and a multi-trophic Rosenzweig-Macarthur model by Hendriks and Mulder (2012).

In general, we chose parameters similar to those used in the PPS literature (Levy et al., 2016; Nevai and Van Gorder, 2012; Shen and Van Gorder, 2017). The nutritional value of prey and subsidy are assumed to be the same and so $\epsilon=\eta=5, \theta=1$. The subsidy decays at rate $\gamma=10$ and the predator dies at rate $\delta=1$. We note that all of these nondimensional parameters have been scaled by the prey growth rate, $r$, so that $\epsilon$ is now the ratio of the predator growth rate due to prey consumption to the prey growth rate, $\eta$ is the same for the predator growth rate to subsidy consumption, and $\delta$ and $\gamma$ are the decay rates of predator and subsidy respectively. These choices correspond to predators which, in a spatially homogeneous setting, would be able to sustain themselves on either prey or subsidy. The biological parameters corresponding to these nondimensional values are within an order of magnitude of some observed populations, such as the zooplankton Daphnia which feeds on marine algae (Murdoch et al., 1998) as well as predation of microtone rodents (Microtus) by larger mustelidae (weasels, Mustela nevalis) (Hanski and Korpimäki, 1995).

\subsection{Qualitative Impact of Delayed Dispersion}

We present examples of qualitatively different impacts of delay on the two-patch separated PPS model. These results are obtained by numerically simulating the system of delaydifferential equations using MATLAB's dde23. In order to obtain accurate results, we reduced the relative error tolerance from 

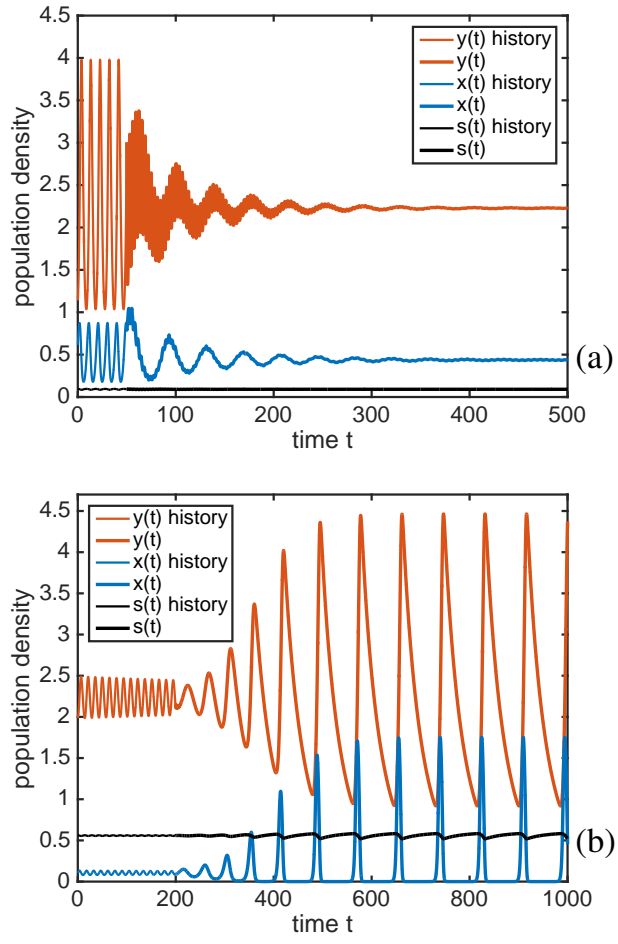

Figure 2: Qualitative change in stability of coexistence equilibrium. In (a), $\kappa=2.4, i=1, \alpha=8, \lambda=1, \tau=3$. The undelayed solution is given as initial history and displays a stable limit cycle about a fixed point. Setting $\tau=3$ at time $t=50$ causes the fixed point to become stable. In (b), $\kappa=3, i=6, \alpha=$ $8, \lambda=0.98, \tau=1$. The undelayed solution is initially oscillatory yet slowly decays to a stable equilibrium. Introducing delay at time $t=200$ induces a stable limit cycle.

the default value $10^{-3}$ to $10^{-9}$ and the maximum step size to $10^{-1}$. The recommended maximum step size for dde23 is a fraction of the period of oscillations of solutions, which was found to be larger than 2 in all simulations performed. Unless otherwise specified, the initial history is taken to be the solution to a system with identical parameter values aside from $\tau$, which is set to zero. This is denoted by "the undelayed solution". Throughout this section, we define $y(t)=y^{(1)}(t)+y^{(2)}(t)$.

Delayed dispersion is largely stabilizing to a two-patch PPS system for small delays, decreasing the amplitude of limit cycles, while increasing the period of oscillations. Both stabilization and period-prolongation effects can be observed in Figure 1 (a)-(c). There is a qualitative change in behavior from Figure 1 (c) to (d), where the oscillations become doubly periodic in $y$ and the amplitude of the limit cycle has increased as the delay is taken to be large. Delayed dispersion may alter the stability of the coexistence equilibrium, as shown in Figure 2. In Figure 2(a), the fixed point undergoes a change in stability and becomes stable as $\tau$ increases from 0 to 3. In Figure 2(b), we observe the opposite; introducing delay causes the fixed point to become unstable. As $\tau$ increases further, the period of the limit cycle increases.

\subsection{Bifurcation Analysis}

We generated several bifurcation diagrams to observe the global effects of the delayed dispersion. We vary $\alpha, \lambda$, and $\tau$ to investigate how access to food resources changes the outcome of the system. We could generate bifurcation diagrams by one of two methods: by running numerical simulations of the system of delay-differential equations for different sets of parameters, or by determining the sign of the major eigenvalue of the modified Jacobian. While the former approach has the benefit of giving more information, it suffers from the slow convergence of solutions and computational expense of delaydifferential equation solving. As generating enough data to reliably produce bifurcation diagrams was time consuming, we opted for a numerical stability analysis.

Regarding changes in the stability of equilibria, we focus our attention on a modification of the two-patch model presented in Nevai and Van Gorder (2012) with delayed dispersion. Here, $\mathbb{P}^{(1)}$ is subsidy-free, $\mathbb{P}^{(2)}$ is prey-free, while the predator is allowed to move between the patches. For simplicity, we assume the rate of dispersal, travel time, and survival rate are equal in both directions: $\alpha^{(1,2)}=\alpha^{(2,1)}=\alpha, \tau^{(1,2)}=\tau^{(2,1)}=\tau$, and $\lambda^{(1,2)}=\lambda^{(2,1)}=\lambda$. Carrying out a standard linearized stability analysis, we obtain a generalized Jacobian. The bifurcation diagrams were generated by finding the eigenvalues of the Jacobian evaluated at relevant fixed points. If the real part of any eigenvalue is greater than zero, the corresponding equilibrium value is unstable. We used the MATLAB opensource package chebfun, whose function roots implements the Marching Squares algorithm for finding roots of bivariate two-dimensional functions.

In Figure 3, we plot bifurcation diagrams in $\tau$ and $\alpha$ for four different values of $\lambda$. The delay may stabilize or destabilize the coexistence equilibrium. However, delayed dispersion is mainly stabilizing for these parameter regimes; for most values of $\alpha$, a limit cycle observed for $\tau=0$ experiences amplitude death upon increasing $\tau$. The equilibrium may again become unstable as $\tau$ is further increased. Destabilization is only observed for low migration rates $\alpha$. As the predator migration survival rate $\lambda$ is decreased from 1 , the system is stabilized. For $\lambda=0.8$, the predator population dies out for sufficiently high migration rates, since a large fraction of the population will die during the frequently occurring migration. The system goes directly from stable coexistence to a predator-free fixed point without experiencing non-equilibrium dynamics. Notice that the existence of a stable predator-free equilibrium is independent of $\tau$ for this parameter regime.

Levy et al. (2016) show that decreasing $\lambda$ from unity could destabilize the coexistence equilibrium of the undelayed twopatch PPS model, causing a limit cycle to appear. The limit cycle would again disappear as $\lambda$ was decreased further and the system would eventually be driven to a predator-free equilibrium. Figure 4 shows how delay impacts this behavior for four different values of dispersion rate $\alpha$. Figure 4(a) has the parameter values used in Levy et al. (2016). Along the $\tau=0$ axis, we see the behavior described above. Increasing $\tau$ destabilizes the coexistence equilibrium at $\lambda=1$. A simulation depicting this change was shown in Figure 2(b). As $\alpha$ decreases, the region of non-equilibrium dynamics shifts to lower $\lambda$-values, and the boundary between stable coexistence and oscillatory behavior becomes more varied. When $\alpha$ becomes sufficiently small, 

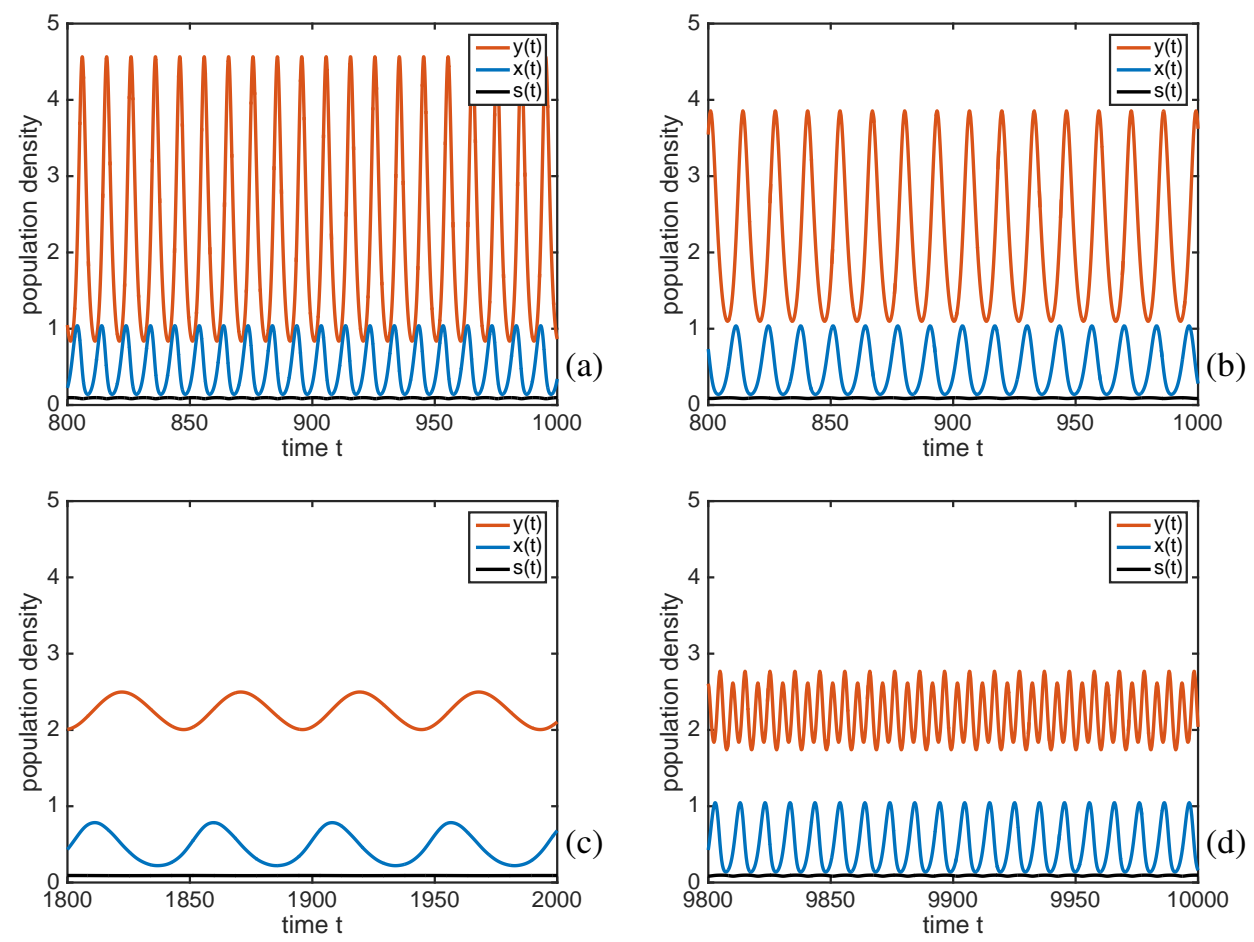

Figure 1: Qualitative change of solution behavior as $\tau$ increases from 0 for a oscillating solution. Parameter values are $\kappa=2.4, i=1, \alpha=8, \lambda=1$. The dispersal delay $\tau$ for (a)-(d) is $0,0.1,3$, and 5 , respectively. The initial history of (b)-(d) is simulation (a).
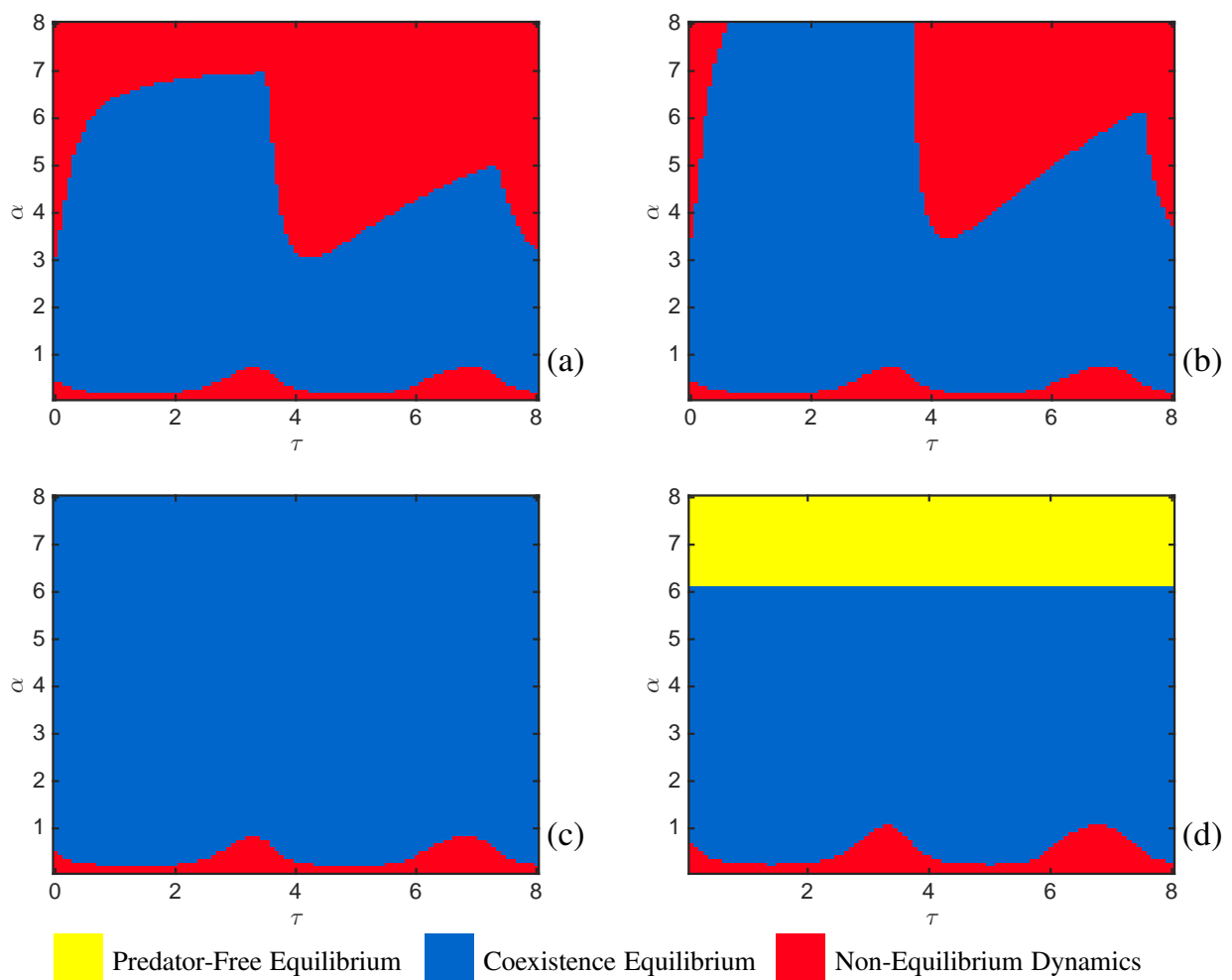

Figure 3: Bifurcation diagram in $\tau, \alpha$ parameter space. $k=2.4, i=1$, varying $\alpha \in[0,8]$ and $\tau \in[0,8]$. The predator migration survival rate $\lambda$ is varied between the plots: Panels (a)-(d) have $\lambda$ equal to $1,0.99,0.95$, and 0.8 , respectively. Non-equilibrium dynamics refer to all dynamics not resulting in equilibria at each node, such as stable limit cycles and less regular oscillations. 

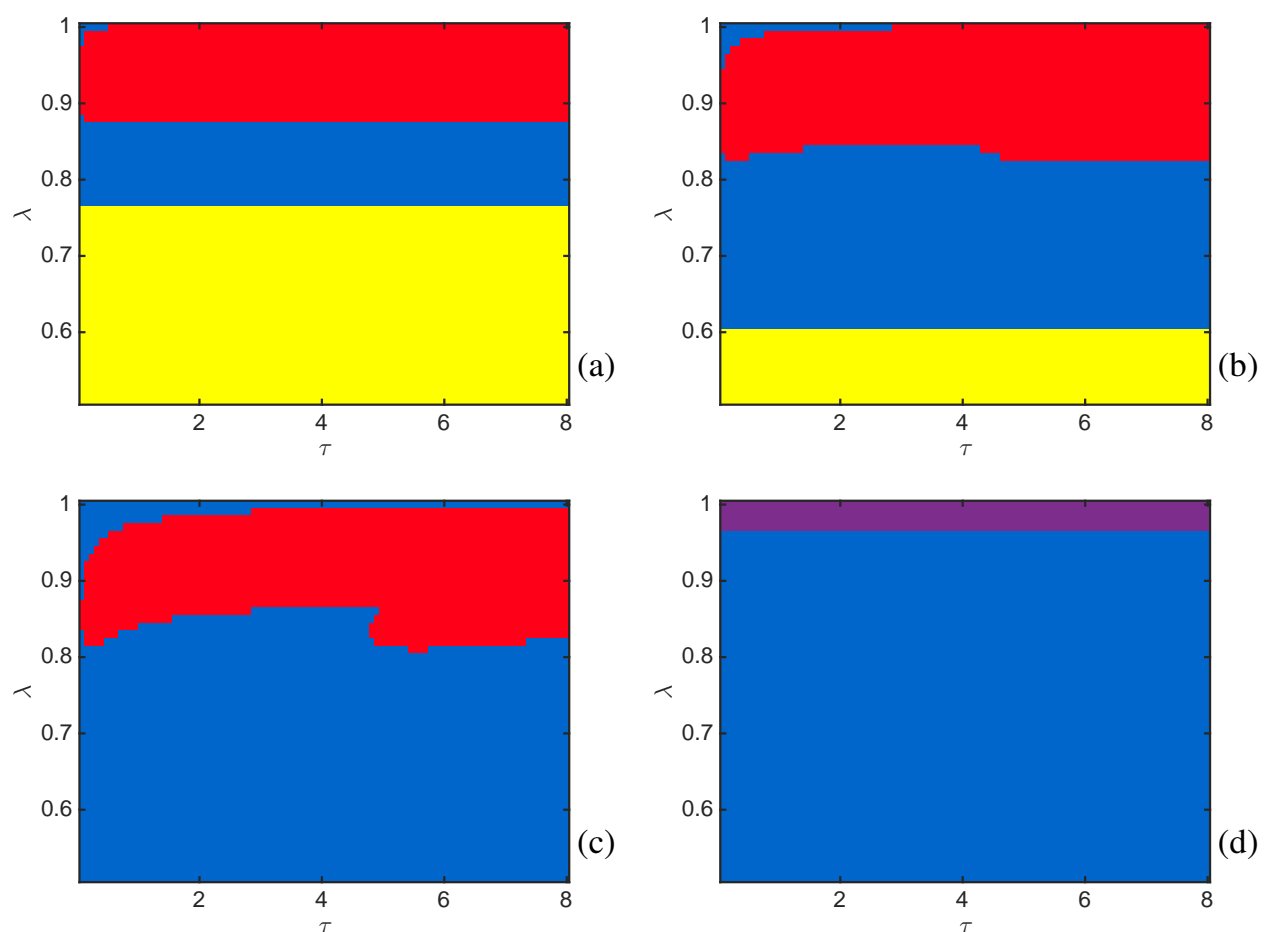

Prey-Free Equilibrium

Predator-Free Equilibrium

Coexistence Equilibrium

Non-Equilibrium Dynamics

Figure 4: Bifurcation diagram in $\tau, \lambda$ parameter space. $k=3, i=6$, varying $\lambda \in[0.5,1]$ and $\tau \in[0,8]$. Panel (a)-(d) has $\alpha=8,5,4$, and 2 , respectively.

all non-equilibrium behavior disappears, and the system shifts directly from stable coexistence to a prey-free equilibrium for high rates of predator survival.

\section{Impact of Network Structure on Dyanmics}

We turn our attention to four patch models to investigate the impact of delay on higher-dimensional systems. Shen and Van Gorder (2017) were able to show that the network structure influences the resulting dynamics of the undelayed PPS. For simplicity, we investigate the four-patch model. While four nodes allow for only small changes in topological structure, it is sufficient to demonstrate the influence that structure has on the dynamics.

An interesting feature of the separated $n$-patch PPS models is the existence of stable equilibria where the prey population dies out on only a subset of the nodes. This cannot occur in the two-patch model, as there is only a single prey population. Hence, for a four-patch separated model with two patches of PP dynamics, there are at least six possible fixed points: subsidy only, predator free, prey free, two fixed points with partial prey extinction, and a positive coexistence equilibrium.

We consider the separated model on the three networks depicted in Figure 5. The first two networks, denoted linear mixed (LM) and linear separated (LS), are both path graphs, but the relative placement of PP and PS systems differ. For the motivating example of the arctic ecosystem, the path graphs could be models of environments such as a string of islands connected by ice. The complete graph (C) simulates an environment along the shore, with two patches on land, and two on the ice.

We performed stability analysis of the three different models (LS), (LM), and (C) by a method similar to what was done in
Section 3. Due to lack of analytic expressions, we used numerical root finding to obtain most of the possible fixed points. The system of characteristic equations for the real and imaginary parts of the eigenvalues of the modified Jacobian was found analytically by use of the MATLAB symbolic math toolbox. This set of equations was then solved using numerical root finding tools. Throughout this and the following section, $y(t)$ is defined as the sum of the predator population across the network.

We produced bifurcation diagrams of the systems in order to get a general idea of the possible behaviors. It became apparent that the differences in the network made comparison between the models challenging. As the focus of our investigation is migration over the network, the underlying structure has a large effect. We performed stability analysis for $\tau$ and $\alpha$, with $\alpha \in[0,4]$ for the path graphs and $\alpha \in[0,2.5]$ for the complete graph. The different ranges of $\alpha$ is to maintain a comparable amount of total migration across the networks.

Figure 6 shows the impact of variation in $\alpha$ and $\tau$ for each model and two different values of $\lambda$. The difference in response to $\alpha$ and $\tau$ across the models is readily observable. The effect of travel time $\tau$ is similar to the two-patch case. For these regions of parameter space, the time delay $\tau$ is only stabilizing. Several bifurcations occur that cause the coexistence fixed point to shift stability. From numerical simulations, we observe the existence of small-amplitude limit cycles near these bifurcations, suggesting that they are supercritical Hopf. Observe that the decrease in $\lambda$ causes almost no change for the (LS) model, but stabilizes both the (LM) and (C) models. In model (C), high migration rates induced prey-death in one patch for $\lambda=1$, which does not occur for $\lambda=0.95$. For the green region in Figure 6(e), we observe both a stable equilibrium with partial prey death, and a 

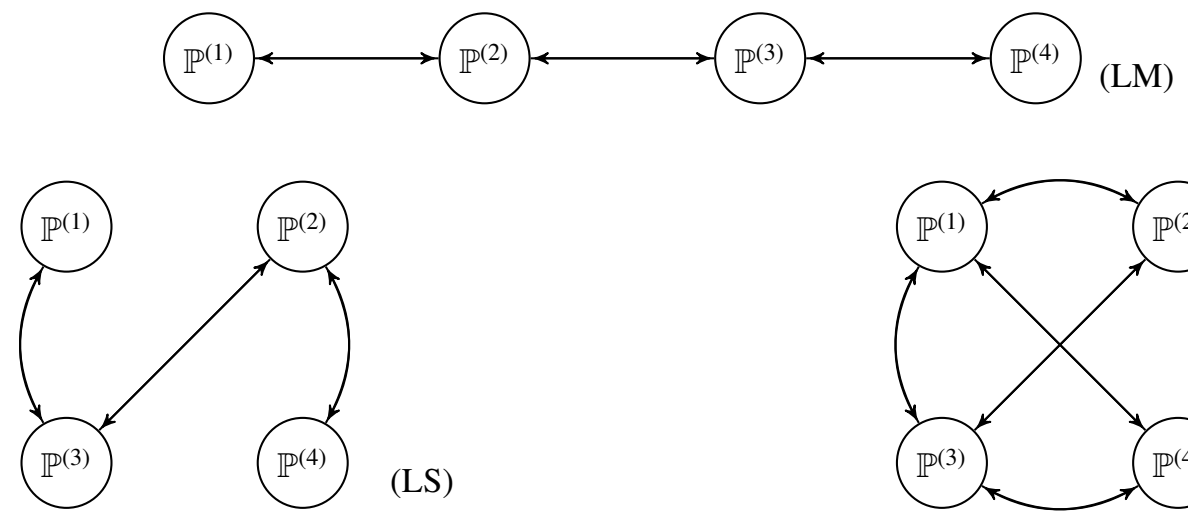

(LS)

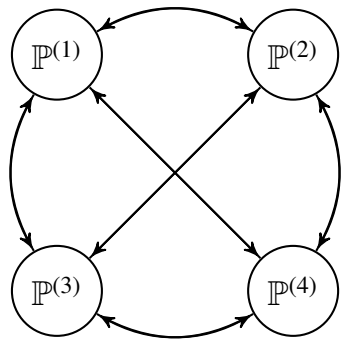

(C)

Figure 5: Schematic of systems under consideration. The separated PPS model on a path graph, where the patches $\mathbb{P}^{(1)}, \mathbb{P}^{(3)}$ are predator-prey $(\mathrm{PP})$ and $\mathbb{P}^{(2)}, \mathbb{P}^{(4)}$ are predator-subsidy (PS) systems. The networks are a linear mixed (LM) graph, a linear separated (LS) graph, and a complete (C) graph, respectively.
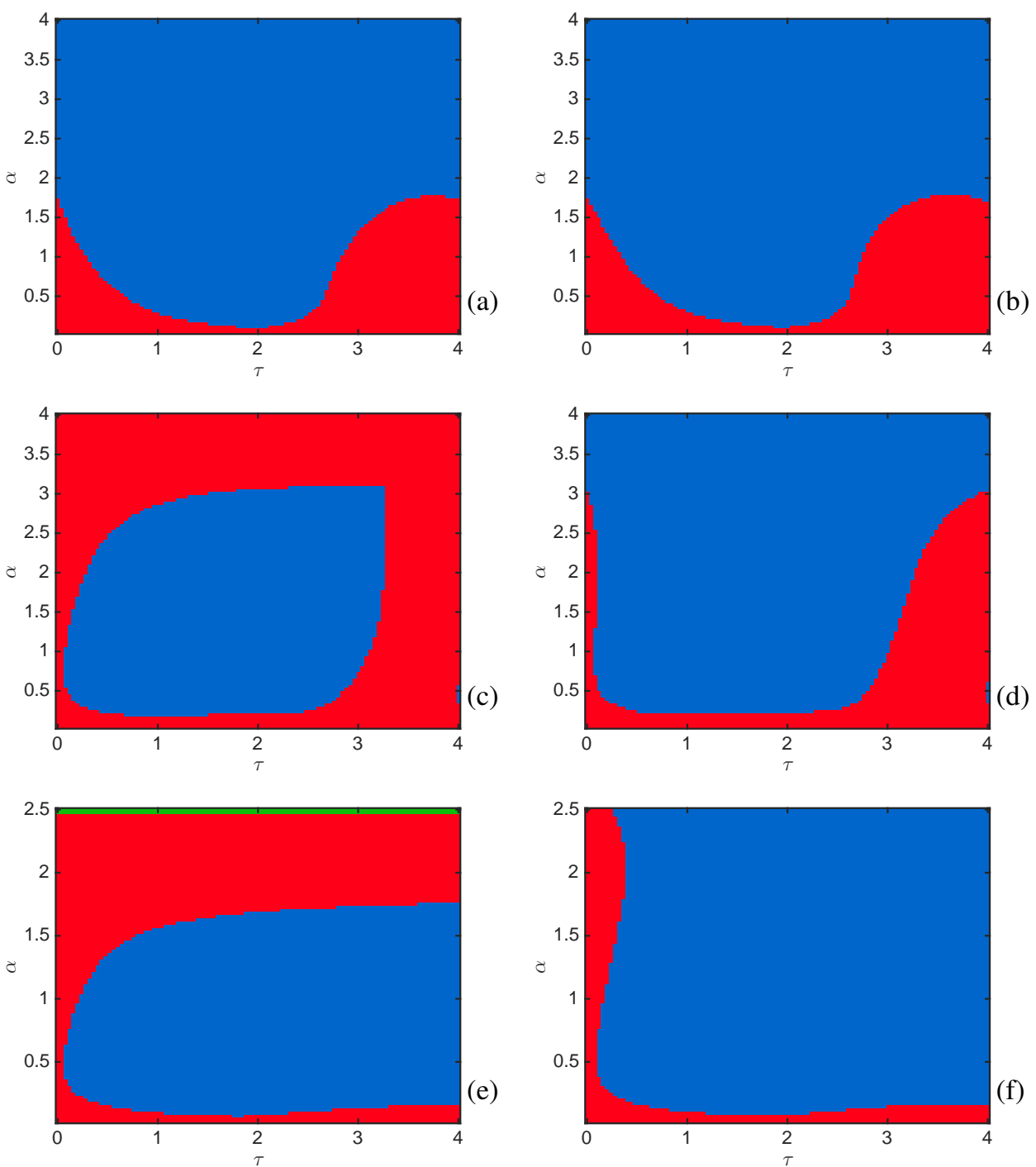

Coexistence Equilibrium

Non-Equilibrium Dynamics

Partial Prey-Death and Non-Equilibrium Dynamics

Figure 6: Bifurcation diagram in $\tau$, $\alpha$ parameter space. Panels (a),(c),(e) have $i=1, \kappa=3, \lambda=1$, while (b),(d),(f) have $i=1, \kappa=3, \lambda=0.95$. (a)-(b), (c)-(d), and (e)-(f) are models (LS), (LM), and (C), respectively. 

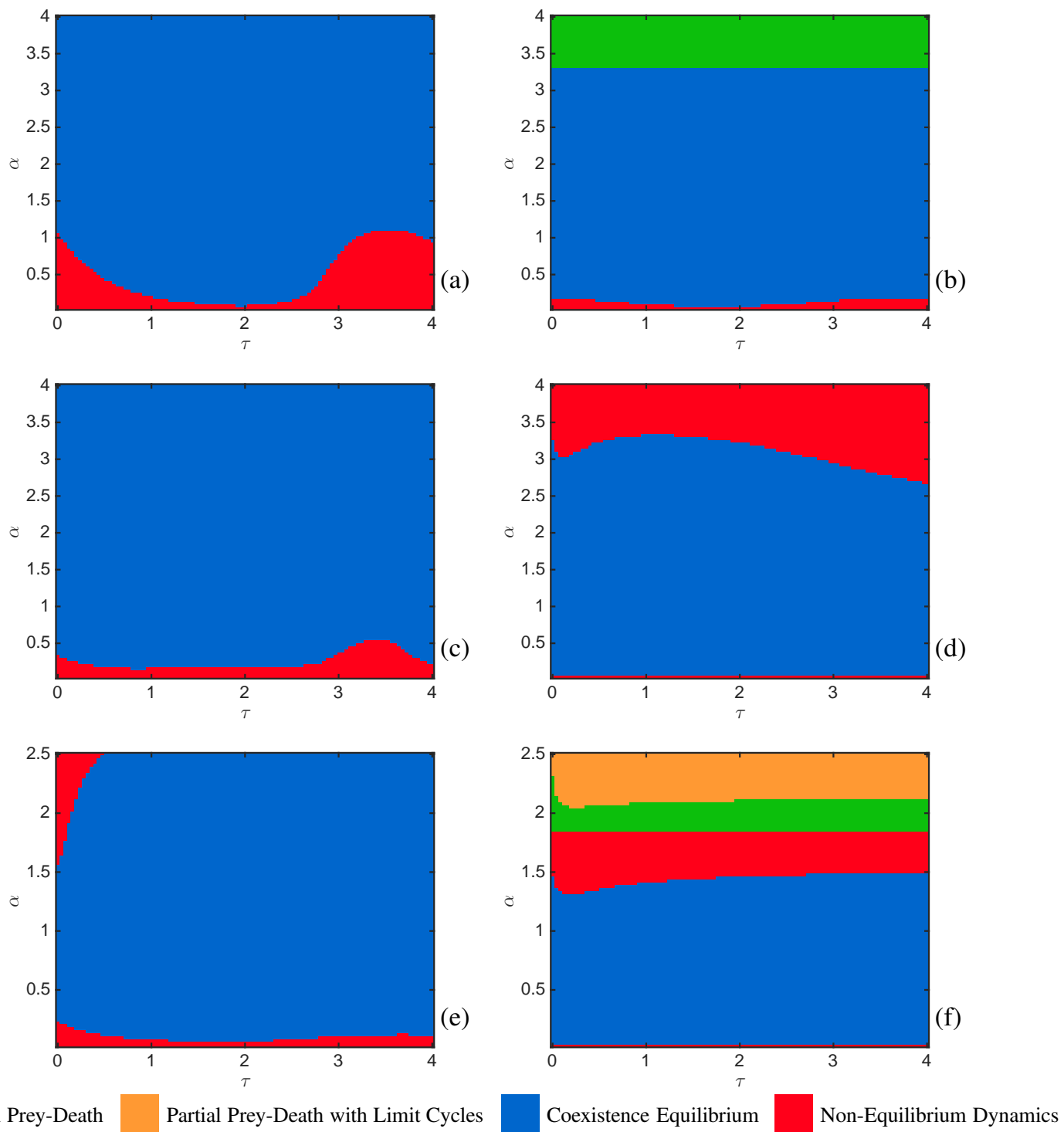

Figure 7: Bifurcation diagram in $\tau, \alpha$ parameter space. Panels (a),(c),(e) have $i=1, \kappa=2.4, \lambda=1$, while (b),(d),(f) have $i=3, \kappa=3, \lambda=1$. (a)-(b), (c)-(d), and (e)-(f) are models (LS), (LM), and (C), respectively.
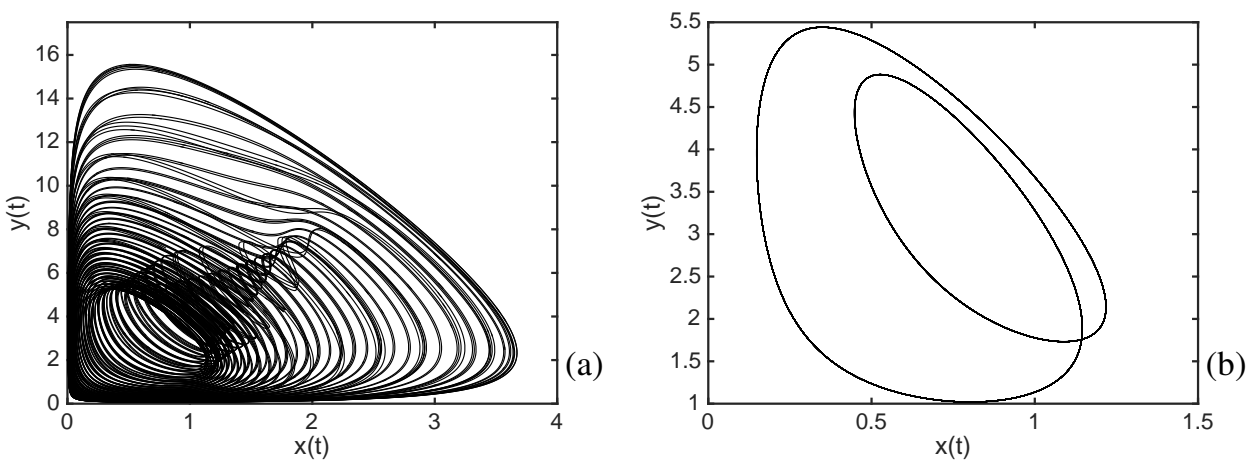

Figure 8: Comparison of the solutions in prey-predator $(x-y)$ space for the (a) undelayed $(\tau=0)$ and $(b)$ delayed $(\tau=0.5)$ solution. Notice that the introduction of delay has severely reduced the amplitude of oscillation, thereby keeping the dynamics bounded further away from zero. Parameters are $\kappa=3, i=1, \lambda=1, \alpha=0.1$. 
stable, positive limit cycle.

Figure 7 depicts the results of stability analysis for two separate sets of parameters. The left column has the same parameters as in Section 3: $i=1, \kappa=2.4$, and $\lambda=1$. The four-patch models appear to be more stable than the two-patch model for this set of parameters. For the complete graph in Figure 7(e), the delay is again stabilizing. In the (LM) model in Figure 7(c), the coexistence equilibrium undergoes destabilization for sufficiently large $\tau$ and $\alpha$ around 0.5 . The right column exhibits behavior not observed in the two-patch model. For models (LM) and $(\mathrm{C})$, increasing delay causes a stable coexistence fixed point to destabilize for a larger range of $\alpha$. Existence of a stable equilibrium with partial prey-death is observed in both models (LS) and (C). For the path graph in Figure 7(b), the prey population goes extinct in $\mathbb{P}^{(3)}$ for $\alpha>3.25$, and this is the only stable attractor in the system. In the complete graph (Figure 7(f)), the PP-patches $\mathbb{P}^{(1)}$ and $\mathbb{P}^{(3)}$ are equal due to symmetry, and hence have equal stability properties. For sufficiently high $\alpha$, a stable equilibrium with partial prey death comes into existence. However, the stable, positive limit cycle originating from the unstable coexistence equilibrium is still an attractor of the system. As $\alpha$ increases further, the equilibrium with partial prey death becomes unstable, and a second stable limit cycle comes into existence. The value of $\alpha$ for which the prey-extinction point destabilizes is dependent on $\tau$.

In the four-patch model, we observe similar behavior to the two-patch case. Increasing $\tau$ from zero can be destabilizing or stabilizing, but stability is again predominant. The four-patch case allows for multistability, and for stable equilibria and limit cycles where one of the two prey-populations is extinct.

\section{Suppression of Quasi-Periodic and Chaotic Dyanamics}

One of the main criticisms of the Rosenzweig-MacArthur model is the existence of the phenomena known as the paradox of enrichment, where an increase in access to food resources may lead a stable system into oscillatory behavior with minima arbitrarily close to zero (Jansen, 1995). As populations are generally observed to maintain a population density away from zero, we desire models that exhibit this behavior.

In the previous two sections, we presented results indicating that introducing delayed dispersion to PPS models could both destabilize and stabilize the dynamics. However, most of the simulations pointed toward stabilization of unstable fixed points. From simulations of the system of delay-differential equations of the two-patch model, we observed that introducing delay could dampen the amplitude of limit cycles. Both are examples of the delay suppressing non-equilibrium dynamics. In this section, we provide evidence of delay suppressing the complexity of the dynamics in systems exhibiting quasi-periodic or chaotic behavior. This is done by applying specific parameter regimes where these behaviors are observed in the undelayed PPS model to our model with delay.

\subsection{Suppressing Quasi-Periodic Dynamics}

A quasi-periodic solution for a dynamical system will oscillate on at least two incommensurate frequencies (Ott, 2002). In an $n$-dimensional system, the attractor is homeomorphic to a $k$-torus, where $2 \leq k<n$ and the solution moves around on this object without ever repeating itself (Strogatz, 2014). It follows that for systems of ordinary differential equations, quasiperiodic behavior can occur when $n \geq 3$. Through adding a delay, we observe that the solutions attain less extreme values when travel time is taken into consideration. Most importantly, the minimum values do not lie close to zero, as illustrated by Figure 8. In this instance, introducing delay induces more regular dynamics.

\subsection{Suppressing Chaos}

Chaotic dynamics have been observed in several predatorprey models, such as a three-species food chain (Hastings and Powell, 1991), a two-patch Rosenzweig-MacArthur predatorprey model (Jansen, 2001), and the non-autonomous PPS model (Levy et al., 2016). Such complex dynamics has not been found in the two-patch autonomous PPS model. In this section, we present evidence of chaotic dynamics in the four-patch (LM) and (LS) models, and observe that introducing delay into these systems has a stabilizing effect.

It is generally challenging to distinguish quasi-periodic behavior from chaotic. The existence of chaotic dynamics in a system can be determined by numerical methods. Estimating Maximal Lyapunov Exponents (MLE) is one such test for chaos (Wolf et al., 1985), based on the theory of Lyapunov exponents of dynamical systems (Strogatz, 2014). The MLE is challenging to implement, and since determining chaos is not the primary concern of this paper, we will use the 0-1 Test (Gottwald and Melbourne, 2004, 2009). The 0-1 Test only requires time series data as input and no knowledge of the system dynamics in real-time. The output is a single number, which in theory is 1 for chaotic time series or 0 for non-chaotic time series. In practice, however, the test will return a number close to 1 for a time series coming from a chaotic attractor and a number close to zero for non-chaotic (Gottwald and Melbourne, 2009). This method is sensitive to oversampling from the time series data and may return an indication of chaos for non-chaotic dynamics. To ensure that our results were correct, we tested different sampling intervals against data we know to be non-chaotic, such as limit cycle solutions and the quasi-periodic solutions from the previous section. As the test is stochastic, we ran the test multiple times and recorded extreme outputs.

Using low migration rates $\alpha$ coupled with high carrying capacities $\kappa$, we were able to find chaotic behavior in the (LM) and (LS) models. The chaos was robust, in that small perturbation of the parameters generated similar behavior. In Figure 9, the solutions of the (LM) model with $i=1, \lambda=1, \kappa=20$, and $\alpha=0.02$ are shown. Figure 9(a),(c) shows the undelayed system, and Figure 9(b),(d) shows the corresponding plots for the system with $\tau=0.5$. For this set of parameters, the result of ten $0-1$ Tests on the undelayed system were all above 0.9970 , indicating chaotic dynamics. The $0-1$ Test for the system with delay returned all values below 0.0853 . When increasing delay from zero to $\tau=0.1$, the dynamics relax onto a stable limit cycle. 

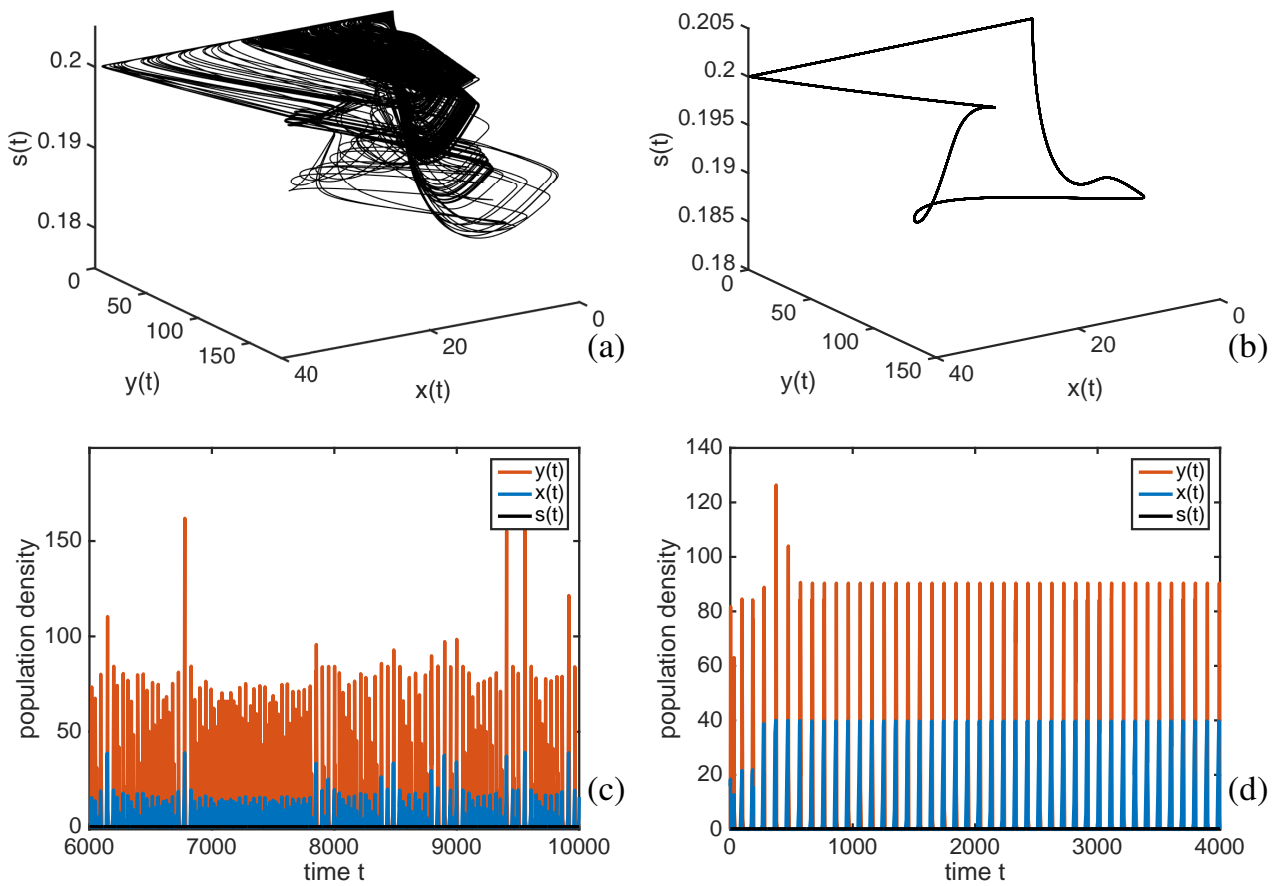

Figure 9: Stabilization of chaos by delay for the (LM) model. Here, $\kappa=20, i=1, \lambda=1, \alpha=0.02$, and $\tau=0$. Panels (a) and (c) illustrate the dynamics of the undelayed $(\tau=0)$ model, while (b) and (d) have non-zero delay $\tau=0.5$. Panel (a) shows the 3D $x-y-s$ phase space for $t \in\left[8.5 \times 10^{3}, 10^{4}\right]$. Panel (b) shows the stable attractor for the system with delay. Panels (c) and (d) show time-series that appear to be indicative of the general behavior.
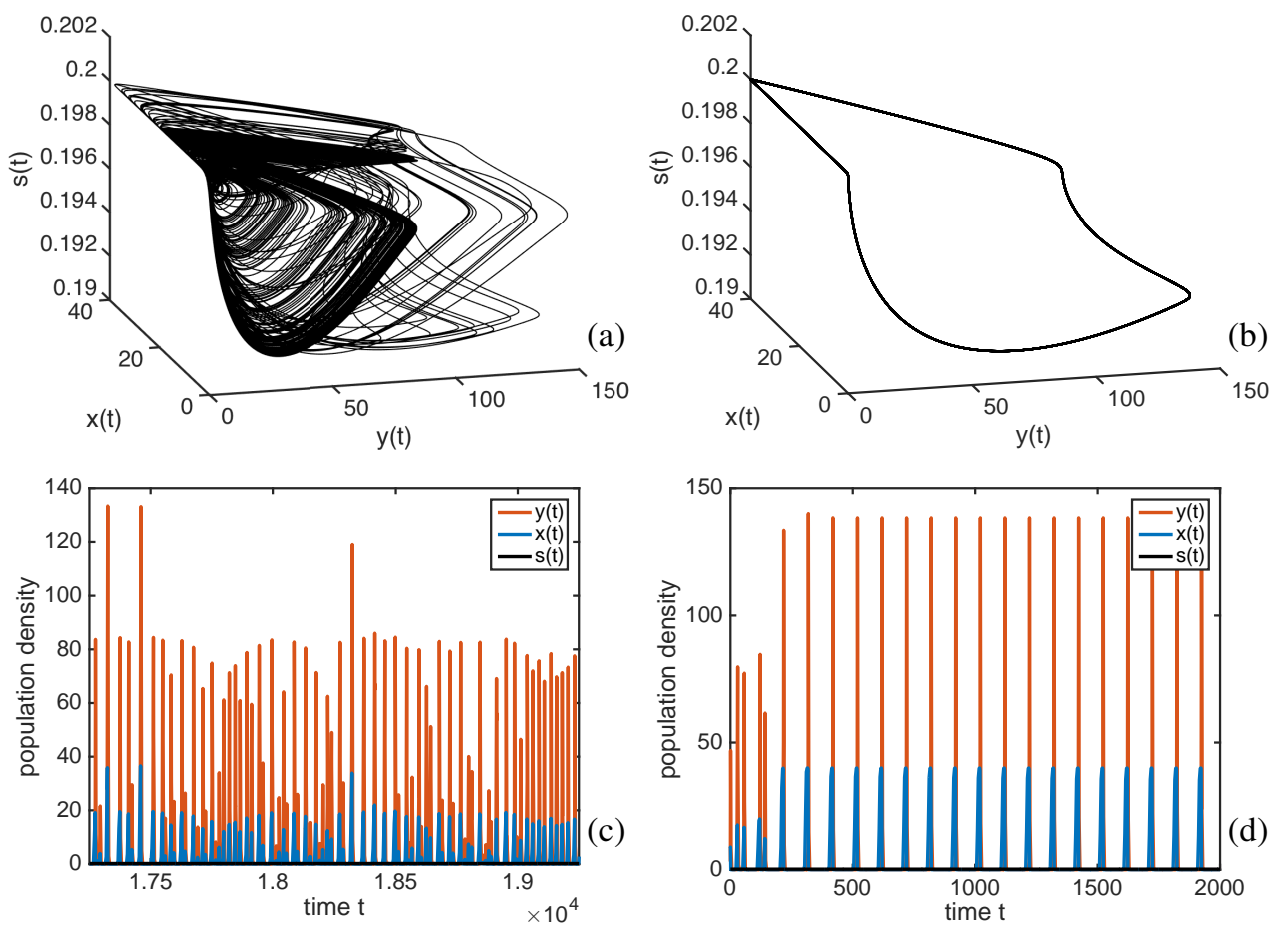

Figure 10: Stabilization of chaos by delay for the (LS) model. Here, $\kappa=20, i=1, \lambda=1, \alpha=0.02$, and $\tau=0$. Panels (a) and (c) illustrate the dynamics of the undelayed $\left(\tau=0\right.$ ) model, while (b) and (d) have non-zero delay $\tau=0.1$. Panel (a) shows the 3D $x-y-s$ phase space for $t \in\left[8.5 \times 10^{3}, 10^{4}\right]$, while panel (b) shows the stable attractor for the system with delay. Panels (c) and (d) show a time-series that appears to be indicative of the general behavior. 
In Figure 10, we show similar results for the (LS) model with equal model parameters. The result of the $0-1$ Test on this system had all values above 0.9974 . Here, we introduced delayed dispersal with $\tau=0.1$, and again observed a relaxation onto a limit cycle. The 0-1 Test for the system with delay returned all values below 0.0341. Note that in Figure 10(c)-(d) we observe an increase in the amplitude of the limit cycle, rather than an increase in the amplitude of the limit cycle as was seen in the (LM) model. This was also the case when running the simulations with $\tau=0.5$ instead of $\tau=0.1$.

\section{Discussion}

The motivating example for this study is the ecological system formed by interaction of Arctic fox, lemming, and seal carrion in the Arctic region. This system differs from the regular predator-prey dynamics due to the foxes' ability to partially or fully sustain themselves on the allochthonous subsidy. Our analysis and results are applicable to other population interactions following the same interaction patterns, such as systems where river otters benefit from a resource subsidy (Ben-David et al., 2005), the wolf, deer, and salmon carcass system (Adams et al., 2010; Darimont et al., 2008), and populations of puma that benefit from mule deer carcass in addition to hunted prey (Bauer et al., 2005). The separated and general $n$-patch models presented in this paper could be good models for these systems, with appropriate choices of parameters describing the living conditions for predator, prey, and subsidy, and sensible choices of underlying network structure, including migration rates and travel time.

This study focused on predator access to food resources: in particular, how implementing non-zero travel time in dispersion affected the dynamics. In the majority of studies done of spatial population models, migration is assumed to be instantaneous. While this assumption may seem appropriate for systems where the travel time between environments is small compared to the time scale of the dynamics within the environments, even small delays have the possibility to greatly change the solution behavior (Wall et al., 2013). The Arctic ecosystem features migration by the fox, both as trips from the shore out on the ice to scavenge for seal carcasses and as quadrennial migration lasting 4-5 months between environments on the shore and on the ice (Wrigley and Hatch, 1976). We do not expect that our choices of parameters pertaining to migration are precise, but hope that they help shine light on phenomena that are lost in the undelayed model.

We examined the effect of introducing constant delayed dispersion in the PPS models. For this implementation of travel time, the location of equilibria of the systems are not impacted by the introduction of delay. This is because our model does not consider the migration survival rate to vary with the length of the journey, a simplification that may be investigated more in future works. However, the behavior of the solutions about the fixed points were found to vary with delay time.

We investigated a two-patch model, finding no instances where the delay caused population death of either the prey or the predator. The delay heavily impacted the stability of a coexistence equilibrium and could both induce limit cycles from a constant, positive solution, or cause a limit cycle solution to experience amplitude death and become a stable coexistence equilibrium. Destabilization was only observed for relatively high subsidy input rates $i$ or low migration rates $\alpha$, while stabilization was found broadly in all other parameter regimes investigated that permitted unstable coexistence equilibria for a range of $\alpha$. Therefore, we concluded that the undelayed and delayed PPS models often fundamentally disagreed in the dynamics of the system, even for small delays. We further found evidence that introducing delays could dampen the amplitude of limit cycles, even when the solution was not forced into a stable equilibrium. Dispersal delay changed the qualitative nature of the oscillatory behavior by decreasing its frequency or by introducing double-amplitude oscillations. In the cases where the delay stabilized the coexistence equilibrium, increasing the time delay $\tau$ further led to destabilization. Hence, a change in travel time may cause changes in the dynamics that only become apparent much later.

We extended our analysis to three different four-patch models and found again that the delay could not force the extinction of any population, but instead had the ability to stabilize or destabilize coexistence equilibria. We found that the structure of the environment greatly changed how variations in access to food sources affected the system. In the four-patch model, there exist coexistence equilibria where a portion of the prey-population dies out locally. Delay may destabilize or stabilize such equilibrium points, but did not force them into or out of existence. Moreover, the delay did not affect the existence of multistability in simulations of the four-patch models, which was previously seen in simulations of corresponding non-delay systems. However, certain quantitative features, such as the types of behaviors possible in multistable regimes, were modified by the inclusion of delay.

As mentioned previously, a main issue with the RosenzweigMacArthur population model is the atto-fox problem, where solutions may fluctuate violently, and reach arbitrarily small values, yet recover (Arditi and Berryman, 1991; Jensen and Ginzburg, 2005), in contrast to what is commonly observed in nature. We found that the stabilizing property of delayed dispersion could regularize the dynamics of the PPS models, hence creating a possible solution to the paradox of enrichment. In Section 5, we showed that introducing small delay into a system exhibiting quasi-periodic solutions with minimum population densities close to zero could force the system onto a limit cycle of much smaller amplitude. We were also able to show that dispersal delay could stabilize chaotic dynamics onto a simple attractor. Although the latter results were for parameter regimes not found in the Arctic ecosystem, it exemplifies that travel time is an important influence on the dynamics of a PPS system.

The importance of these results to a mathematical biologist is that travel time in a system with migratory populations has the ability to greatly change the dynamics of the system, even for small delays. Hence, the assumption of instantaneous migration should not be made lightly. Additionally, changes to the delay in travel between patches may push a stable equilibrium point 
into large oscillations; for example, see Figure 2(b). This may be caused both by increases and decreases in the delay. While we might intuitively think that decreasing travel time will benefit the predator population by increasing access to food sources, this may in fact destabilize the system and leave the populations susceptible to extinction. Moreover, we showed that the qualitative behaviour of four-patch models could differ greatly with the same general parameter set due to differences in the migration network. Hence, changing the underlying network structure by restricting or offering access to the prey could have complex and unforeseen consequences.

\section{Conclusions and future work}

We proposed a model motivated by the Arctic ecosystem, where migratory foxes are able to sustain themselves on both hunted lemming or scavenged seal carcasses. We investigated how access to food resources, especially the travel time, impacted the stability and behaviour of the predator-prey-subsidy system. We concluded that dispersal delay is primarily stabilizing with respect to pre-existing non-equilibrium dynamics (such as limit cycles), which aligns well with earlier results from predator-prey systems (Klepac et al., 2007; Neubert et al., 2002; Zhang et al., 2015b). However, we also showed that delay can destabilize the predator-prey dynamics by inducing limit cycles from coexistence equilibria in the corresponding undelayed model. These results were found for both the twopatch and a range of four-patch models. As we found that applying delay in migration to an undelayed system can stabilize quasi-periodic and chaotic dynamics, incorporating travel time might fundamentally change the dynamics of a system. The stabilizing property of dispersion delay, where limit cycle solutions collapse to coexistence equilibria and solutions exhibiting chaotic dynamics are reduced to limit cycles, establishes the incorporation of travel time as a possible resolution to the paradox of enrichment.

In our motivating example of the Arctic ecosystem, the predator takes short trips out on the ice to access the resource subsidy, and migrates every three to four years, when it covers up to 1000km (Wrigley and Hatch, 1976). A more realistic analysis of this system could involve differentiating these travels by implementing dispersion delays of different order across the network, and studying the effect of this on solution behavior. Scavenging trips out on the sea ice during the warmer seasons may be more challenging and hazardous for the fox to complete, and hence of longer duration in the summer than in the winter. Therefore, there is an argument for making the travel delay seasonally dependent by implementing non-constant time delay, $\tau(t)$. This would be an extension of the work done on seasonality by Levy et al. (2016) and should be implemented along with time-dependent migration rate and migration survival rate, $\alpha(t)$ and $\lambda(t)$. There is evidence that the migration patterns of the Arctic fox align with the cyclic behavior of the lemming population, in that the migration rates of the fox population will increase when lemming is scarce (Angerbjorn et al., 1999; Dalén et al., 2004; Gilg and Yoccoz, 2010). By including density-dependent migration rates for the predator, one might incorporate this effect.

Finally, we assumed that the temporal delays were discrete. This is equivalent to assuming the travel time is equal for every traveller, on every trip taken. A more realistic model would only assume a distribution of travel times. This implementation of delayed dispersion, which also takes into account that increased travel time implies decreased migration survival rate, was investigated for a one-patch Rosenzweig-MacArthur model by Klepac et al. (2007) and could be extended to multi-patch PPS models, such as those we considered here.

\section{A. Existence and Boundedness of Solutions to (15)-(19)}

In order to ensure that solutions to the model (15)-(19) are biologically feasible, we now prove that solutions exist and remain bounded.

\section{A.1. Existence of a Continuous Solution}

We show that solutions to our models exist and are continuous. This result is used to prove that the model solutions are non-negative and bounded above for physical initial data. For the following proofs, we will need to ensure that the solution to the relevant system of delay-differential equations exists and is continuous. We will here give a result due to Driver (1963).

Let $m, n \geq 1$, with $m, n \in \mathbb{Z}$ and $\mathbf{u}(t): \mathbb{R}^{+} \mapsto \mathbb{R}^{n}$, where $\mathbb{R}^{+}$is the positive real line. For $k=1, \ldots, m$, let $g_{k}(t, \mathbf{u}(t))$ : $\mathbb{R}^{+} \times \mathbb{R}^{n} \mapsto \mathbb{R}^{+}$, hence $g_{k}$ is real-valued and non-negative. Let $F: \mathbb{R}^{+} \times \mathbb{R}^{n} \times \mathbb{R}^{m n}$ define a delay-differential equation of the form

$$
\frac{d}{d t} \mathbf{u}(t)=\mathbf{F}\left(t, \mathbf{u}(t), \mathbf{u}\left(t-g_{1}(t, \mathbf{u}(t))\right), \ldots, \mathbf{u}\left(t-g_{m}(t, \mathbf{u}(t))\right)\right) .
$$

Equation (25) is a $n$-dimensional delay-differential equation with $m$ delayed terms. Each delay $g_{k}(t, \mathbf{u}(t))$ may be state and time dependent. Let $t_{0} \geq 0$ be the initial time and $\phi(t)=$ $\left(\phi_{1}(t), \ldots, \phi_{n}(t)\right)$ be an initial history for $t \in\left(-\infty, t_{0}\right]$. The following Theorem is found in Winston (1971) and is proven in Driver (1963).

Theorem 1: Local Existence of Solution. Let $\mathbf{F}$ be as given above and locally continuous. Let $g_{k}$ be continuous for all $t \geq t_{0}, \mathbf{u} \in \mathbb{R}^{n}$ for $k=1, \ldots, m$, and let $\phi(t)$ be continuous for $t \in\left(-\infty, t_{0}\right]$. Then there exist $\varepsilon>0$ and a function $\mathbf{u}(t)$ such that $\mathbf{u}(t)$ satisfies Equation (25) for $t_{0} \leq t<t_{0}+\varepsilon$.

We note that state and time dependent delay is not required for the models presented in this paper; however, it can be useful for possible extensions. Theorem 1 implies that a continuous solution $\mathbf{u}(t)$ exists for all values of $t, \mathbf{u}(t)$ such that $\mathbf{F}$ is continuous. For the non-dimensional models presented in this paper, $\mathbf{F}$ is continuous whenever $s^{(j)}, x^{(j)} \neq-1$ for all patches $\mathbb{P}^{(j)}$. As we will show in the following Section A.2, this is not attainable for non-negative and continuous initial histories. Hence, by a boot-strapping argument, global solutions exist for all models presented in this paper, assuming physically realistic initial data. 


\section{A.2. Dynamics Bounded Below}

The models presented in this paper describe physical quantities that cannot be negative; the population density of an animal population or the available amount of a resource must not go below zero. In this section, we will prove that the dynamics of the separated $n$-patch model are non-negative. This is an important result to ensure the feasibility of our model.

Lemma 1: Dynamics Bounded Below. Consider the separated $n$-patch model with non-negative parameters as given by Equations (15)-(19). Let the system have non-negative initial conditions $x^{(\ell)}(0)=x_{0}^{(\ell)}$ for $\mathbb{P}^{(\ell)} \in \mathcal{N}_{X}, s^{(k)}(0)=s_{0}^{(k)}$ for $\mathbb{P}^{(k)} \in \mathcal{N}_{S}$ and a continuous, non-negative initial history $y^{(j)}(t)=y_{0}^{(j)}(t)$ for $t \in\left[-\max _{\ell \neq j} \tau^{(\ell, j)}, 0\right]$, for all $\mathbb{P}^{(j)}$. Then $x^{(\ell)}(t) \geq 0, s^{(k)}(t) \geq 0$, and $y^{(j)}(t) \geq 0$ for all $\ell$ such that $\mathbb{P}^{(\ell)} \in \mathcal{N}_{X}, k$ such that $\mathbb{P}^{(k)} \in \mathcal{N}_{S}$, and all $j \in\{1, \ldots, n\}$, for all time $t$.

Proof: By Theorem 1, a continuous solution to the described system exists for some time $t \in[0, \varepsilon), \varepsilon>0$. We will prove that this solution is bounded below by zero by considering the movement of the $x, s$, and $y$ components separately.

Consider the dynamics of $x^{(\ell)}$ given by Equation (15). For $x^{(\ell)}$ to be negative at some time $t$, there must exist a time $\bar{t}$ such that $x^{(\ell)}(\bar{t})=0$ by the Intermediate Value Theorem. However, when $x^{(\ell)}=0, d x^{(\ell)} / d t=0$, and so $x^{(\ell)}(t)=0$ for all $t \geq \bar{t}$. Hence $x^{(\ell)}$ may not be negative. Similarly, consider the case that $s^{(k)}(t)<0$ for some time. By the same argument, we require that $s^{(k)}(\bar{t})=0$ for some time $\bar{t}$. By Equation (13), $d s^{(k)} / d t(\bar{t})=$ $i^{(k)} \geq 0$. Hence $s^{(k)} \geq 0$ for all $t$.

Lastly, consider the case that $y^{(j)}<0$ for some $\mathbb{P}^{(j)}$. Let $T$ denote the set of times where $y^{(j)}<0$ for some $j: T=$ $\left\{t \geq 0 \mid \exists j\right.$ with $\left.y^{(j)}(t)<0\right\}$. If $T \neq \varnothing, T$ must have an infimum $\bar{t}$. At $t=\bar{t}, y^{(j)}(t) \geq 0$ for all $j$, and there exists an $i$ such that $y^{(i)}(\bar{t})=0$ and $d y^{(i)} / d t<0$ at $\bar{t}$. Assume without loss of generality that $i$ is such that $\mathbb{P}^{(i)} \in \mathcal{N}_{X}$. By Equation (16), we have

$$
\frac{d y^{(i)}}{d t}(\bar{t})=\sum_{\ell \neq i} \lambda^{(\ell, j)} \alpha^{(\ell, i)} y^{(\ell)}\left(\bar{t}-\tau^{(\ell, i)}\right)
$$

As $\bar{t}$ is the onset of negativity, $y^{(\ell)}\left(\bar{t}-\tau^{(\ell, i)}\right) \geq 0$ for all $\ell$, and so $d y^{(i)} / d t \geq 0$ at $\bar{t}$. Hence we have a contradiction, and $y^{(j)} \geq 0$ for all $t$.

This result can easily be extended to the general $n$-patch PPS model.

\section{A.3. Dynamics Bounded Above}

The solutions of a physical model of population behavior must be bounded below to be relevant. Likewise, it is pertinent that solutions are bounded above, since we do not want unbounded populations. Hence, we offer a boundedness result for the separated $n$-patch model. We state the following global existence result that can be derived from a bootstrapping argument from Theorem 1 and Lemma 1:

Corollary 1: Global Existence of Solution with Physical Initial Data. Consider the separated $n$-patch model with non-negative parameters as given by Equations (15)-(19). Let the system have non-negative initial conditions $x^{(\ell)}(0)=x_{0}^{(\ell)}$ for $\mathbb{P}^{(\ell)} \in \mathcal{N}_{X}$, $s^{(k)}(0)=s_{0}^{(k)}$ for $\mathbb{P}^{(k)} \in \mathcal{N}_{S}$ and a continuous, non-negative initial history $y^{(j)}(t)=y_{0}^{(j)}(t)$ for $t \in\left[-\max _{\ell \neq j} \tau^{(\ell, j)}, 0\right]$, for all $\mathbb{P}^{(j)}$. Then, the solutions of the system are globally continuous.

At a time $t$, there are two possibilities for the relationship between $y^{(j)}(t)$ and $y^{(j)}\left(t-\tau^{(j, \ell)}\right)$ : either $y^{(j)}(t) \geq y^{(j)}\left(t-\tau^{(j, \ell)}\right)$, or $y^{(j)}(t)<y^{(j)}\left(t-\tau^{(j, \ell)}\right)$. Hence, we can partition the time $t>0$ by considering all possible configurations of $\mathbf{y}(t)=$ $\left(y^{(1)}(t), \ldots, y^{(n)}(t)\right)$ relative to $\mathbf{y}\left(t-\tau^{(j, \ell)}\right)$, for each pair $(j, \ell)$. As it will be necessary to keep track of these configurations for the boundedness lemma and proof, we introduce the following time-dependent sets

$S(t)=\left\{(j, \ell): j, \ell \in\{1, \ldots, n\}, j \neq \ell\right.$, and $\left.y^{(j)}(t)<y^{(j)}\left(t-\tau^{(j, \ell)}\right)\right\}$,
$\bar{S}(t)=\left\{(j, \ell): j, \ell \in\{1, \ldots, n\}, j \neq \ell\right.$, and $\left.y^{(j)}(t) \geq y^{(j)}\left(t-\tau^{(j, \ell)}\right)\right\}$.

It is clear that $S(t) \cup \bar{S}(t)=\{1, \ldots, n\} \times\{1, \ldots, n\}$ for all $t$. For ease of notation, let $S_{j}(t)=\{j:(j, \ell) \in S(t)\}$, and similarly $\bar{S}_{j}(t)=\{j:(j, \ell) \in \bar{S}(t)\}$.

Each delay $\tau^{(j, \ell)}$ introduces two possibilities, and so it follows that the number of possible time partition sets is $2^{n(n-1)}$. Giving a full enumeration of these sets is unnecessary for the statement and proof of a boundedness result, as we can consider them generally.

Lemma 2: Dynamics Bounded Above for the n-Patch Separated System. Consider the separated $n$-patch PPS model with non-negative parameters as previously described, predator death rate $\delta^{(j)}>0$ for all $j$, and subsidy removal rate $\gamma^{(k)}>0$ for all $\mathbb{P}^{(k)} \in \mathcal{N}_{S}$. Let the system have non-negative initial conditions $x_{0}^{(\ell)}$ for $\mathbb{P}^{(\ell)} \in \mathcal{N}_{X}, s_{0}^{(k)}$ for $\mathbb{P}^{(k)} \in \mathcal{N}_{S}$ and a nonnegative, continuous, and bounded initial history $y^{(j)}(t)=y_{0}^{(j)}(t)$ for $t \in\left[-\max _{\ell \neq j} \tau^{(\ell, j)}, 0\right]$, for all $\mathbb{P}^{(j)}$. Assume that $y^{(j)}(t)>0$ for all $\mathbb{P}^{(j)}$. Moreover, assume

$$
\delta^{(j)}+\sum_{\ell \in S_{j}} \alpha^{(j, \ell)}\left(1-\lambda^{(j, \ell)} K^{(j, \ell)}(t)\right) \geq 0
$$

for each $j$ such that $(j, \ell) \in S(t)$, where $S$, and $S_{j}$ are as defined above, and where $K^{(j, \ell)}(t)$ is such that $K^{(j, \ell)}(t) y^{(j)}(t)=y^{(j)}\left(t-\tau^{(j, \ell)}\right)$. Then $x^{(\ell)}(t), s^{(k)}(t)$ and $y^{(j)}(t)$ are bounded for all $\ell$ such that $\mathbb{P}^{(\ell)} \in \mathcal{N}_{X}, k$ such that $\mathbb{P}^{(k)} \in \mathcal{N}_{S}$, and all $j \in\{1, \ldots, n\}$, for all time $t$.

Proof: By Corollary 1, the solution to the described system is continuous for $t \geq 0$. As in Lemma 2.1, the solutions $x^{(\ell)}(t), s^{(k)}(t)$ for $\ell$ such that $\mathbb{P}^{(\ell)} \in \mathcal{N}_{X}, k$ such that $\mathbb{P}^{(k)} \in \mathcal{N}_{S}$ are bounded above.

We will prove that $y^{(j)}(t)$ is bounded above under the given assumptions by considering a general time $t$, assuming $\mathbf{y}$ has previously been bounded. As the solutions are continuous, there must be a $t$ for which this assumption holds. For this time, we have sets $S, S{ }_{j}, \bar{S}$, and $\bar{S}_{i}$ as described above. Adopt the notation $j \in S$ to signify $(j, \ell) \in S$ for some $\ell$. Note that 
some of these sets may be empty, but they must fulfill the following relations: $S \cup \bar{S}=\{1, \ldots, n\} \times\{1, \ldots, n\}, S \cap \bar{S}=\varnothing$, $S_{j} \cup \bar{S}_{j}=\{1, \ldots, n\}$, and $S_{j} \cap \bar{S}_{j}=\varnothing$. If $\bar{S}=\varnothing, y^{(j)}(t)$ is bounded by its past values, and $t$ must pass into another partition before the solution can grown unbounded. Hence, we assume that $S \neq \varnothing$. For the same reason, we will also assume that there is at least one $j$ such that $j \notin S$.

Define the quantity $B$ as follows:

$$
\frac{d B}{d t}(t)=\sum_{j_{X} \in \mathcal{N}_{X}} \epsilon^{\left(j_{X}\right)} x^{\left(j_{X}\right)}(t)+\sum_{j_{S} \in \mathcal{N}_{S}} \frac{\eta^{\left(j_{S}\right)}}{\phi^{\left(j_{s}\right)}} s^{\left.j_{x}\right)}+\sum_{j \in\{1, \ldots, n\}} y^{(j)}(t) .
$$

Taking the derivative of $B$ with respect to time, and using similar manipulations as what was done in the proof of Lemma 2.1, we get

$$
\begin{aligned}
& \frac{d B}{d t}(t) \leq \sum_{j \in\{1, \ldots, n\}} C_{j}-\delta^{(j)} y^{(j)}(t) \\
& \quad+\sum_{\ell \neq j} \alpha^{(\ell, j)}\left[\left(\lambda^{(\ell, j)} y^{(\ell)}\left(t-\tau^{(\ell, j)}\right)-y^{(j)}(t)\right]\right. \\
& \leq \tilde{C}+\sum_{i \in \bar{S}}\left[-\bar{\delta}^{(i)} y^{(i)}(t)+\sum_{k \in \bar{S}_{i}} \alpha^{(i, k)}\left[\lambda^{(i, k)} y^{(i)}\left(t-\tau^{(i, k)}\right)-y^{(i)}(t)\right]\right] \\
& \quad+\sum_{j \in S}\left[-\delta^{(j)} y^{(j)}(t)+\sum_{\ell \in S_{j}} \alpha^{(j, \ell)}\left[\lambda^{(j, \ell)} y^{(j)}\left(t-\tau^{(j, \ell)}\right)-y^{(j)}(t)\right],\right.
\end{aligned}
$$

where $C_{j}$ bounds the $y$-independent terms of $\mathbb{P}^{(j)}$, and $\tilde{C}$ is the sum of these terms. $\bar{\delta}^{(i)}$ is defined as zero if $i \in S$, and $\delta^{(i)}$ if $i \notin S$, and is defined to avoid over counting of negative terms. It follows from assumption that $\bar{\delta}^{(i)}>0$ for at least one $i \in \bar{S}$.

Using the relations of set $S$ and $\bar{S}$, the following substitutions can be made

$$
\begin{aligned}
\frac{d B}{d t}(t) \leq \tilde{C} & -\sum_{i \in \bar{S}}\left[\bar{\delta}^{(i)}+\sum_{k \in \bar{S}_{i}} \alpha^{(i, k)}\left(1-\lambda^{(i, k)}\right)\right] y^{(i)}(t) \\
& -\sum_{j \in S}\left[\delta^{(j)}+\sum_{\ell \in S_{j}} \alpha^{(j, \ell)}\left[1-\lambda^{(j, \ell)} K^{(j, \ell)}(t)\right] y^{(j)}(t) .\right.
\end{aligned}
$$

As $y^{(j)}(t)$ is bounded by its past value for all $j$, it cannot have grown unbounded for the present $t$, and hence we do not need to bound $y^{(j)}(t)$ for this partition of time. By the restriction given in the statement of Lemma 2, the outer sum in $j$ on the second line of (28) is negative, and so we may remove it from the bound. Define $\xi^{(i)}$ such that

$$
\xi^{(i)}=\bar{\delta}^{(i)}+\sum_{k \in \bar{S}_{i}}\left(1-\lambda^{(i, k)}\right) \alpha^{(i, k)}
$$

for $i \in \bar{S}$. Recall that $\lambda \leq 1$ and the definition of $\bar{\delta}^{(i)}$. Hence, $\xi^{(i)} \geq 0$ for all $i$, and $\xi^{(i)}>0$ for $i \notin S$. By assumption, there exists $i$ such that $\xi^{(i)}>0$. If $\xi^{(i)}=0, y^{(i)}(t)$ is bounded by its past history, and need not be bounded. Define $\xi=\min \left\{\xi^{(i)}\right.$ : $\left.\xi^{(i)}>0\right\}$. The inequality (28) then becomes

$$
\frac{d B}{d t}(t) \leq \tilde{C}-\xi\left[\sum_{i \in \bar{S}} y^{(i)}(t)\right] .
$$

Assume now that some $y^{(i)}$ for $i \in \bar{S}$ grows unbounded. Then $y^{(i)}$ must at some time be larger than some of its delayed terms. Thus, $i \in \bar{S}$ for some $t$, for which it is arbitrarily large. Therefore, $\sum_{i \in \bar{S}} y^{(i)}(t)>\tilde{C} / \xi$, as all components $y^{(j)}>0$. Hence $d B / d t<0$, and $B(t)$ must be decreasing. Notice that this bound is independent of all solutions bounded in this partition set. Hence, $y^{(i)}$ cannot grow arbitrarily large for any patch, and so the solution must be bounded.

We have not observed unbounded solutions for any numerical simulations. We believe the restrictions on the growth rate, given by (27), could be lifted, but we do not pursue this technicality here.

\section{References}

Adams, L. G., Farley, S. D., Stricker, C. A., Demma, D. J., Roffler, G. H., Miller, D. C., Rye, R. O., 2010. Are inland wolf-ungulate systems influenced by marine subsidies of pacific salmon? Ecological Applications 20 (1), 251-262.

Angerbjorn, A., Tannerfeldt, M., Erlinge, S., 1999. Predator-prey relationships: arctic foxes and lemmings. Journal of Animal Ecology 68 (1), 34-49.

Arditi, R., Berryman, A. A., 1991. The biological control paradox. Trends in Ecology \& Evolution 6 (1), 32.

Bassett, A., Krause, A. L., Van Gorder, R. A., 2017. Continuous dispersal in a model of predator-prey-subsidy population dynamics. Ecological Modelling 354, 115-122.

Bauer, J. W., Logan, K. A., Sweanor, L. L., Boyce, W. M., 2005. Scavenging behavior in puma. The Southwestern Naturalist 50 (4), 466-471.

Ben-David, M., Blundell, G. M., Kern, J. W., Maier, J. A., Brown, E. D., Jewett, S. C., 2005. Communication in river otters: creation of variable resource sheds for terrestrial communities. Ecology 86 (5), 1331-1345.

Choudhury, S. R., 1992. On bifurcations and chaos in predator-prey models with delay. Chaos, Solitons \& Fractals 2 (4), 393-409.

Dalén, L., Fuglei, E., Hersteinsson, P., Kapel, C. M., Roth, J. D., Samelius, G., Tannerfeldt, M., Angerbjörn, A., 2004. Population history and genetic structure of a circumpolar species: the arctic fox. Biological Journal of the Linnean Society 84 (1), 79-89.

Darimont, C. T., Paquet, P. C., Reimchen, T. E., 2008. Spawning salmon disrupt trophic coupling between wolves and ungulate prey in coastal british columbia. BMC ecology 8 (1), 14.

Driver, R. D., 1963. Existence theory for a delay-differential system. Contributions to Differential Equations 1, 317-336.

Gilg, O., Yoccoz, N. G., 2010. Explaining bird migration. Science 327 (5963), 276-277.

Gilpin, M. E., Rosenzweig, M., 1972. Enriched predator-prey systems: theoretical stability. Science 177 (4052), 902-904.

Goldwyn, E. E., Hastings, A., 2009. Small heterogeneity has large effects on synchronization of ecological oscillators. Bulletin of mathematical biology 71 (1), 130-144.

Gottwald, G. A., Melbourne, I., 2004. A new test for chaos in deterministic systems. In: Proceedings of the Royal Society of London A: Mathematical, Physical and Engineering Sciences. Vol. 460. The Royal Society, pp. 603611.

Gottwald, G. A., Melbourne, I., 2009. On the implementation of the $0-1$ test for chaos. SIAM Journal on Applied Dynamical Systems 8 (1), 129-145.

Halaj, J., Wise, D. H., 2002. Impact of a detrital subsidy on trophic cascades in a terrestrial grazing food web. Ecology 83 (11), 3141-3151.

Hanski, I., Korpimäki, E., 1995. Microtine rodent dynamics in northern europe: parameterized models for the predator-prey interaction. Ecology 76 (3), 840-850.

Hastings, A., Powell, T., 1991. Chaos in a three-species food chain. Ecology 72 (3), 896-903.

Hauzy, C., Gauduchon, M., Hulot, F. D., Loreau, M., 2010. Density-dependent dispersal and relative dispersal affect the stability of predator-prey metacommunities. Journal of Theoretical Biology 266 (3), 458-469.

Henden, J.-A., Ims, R. A., Yoccoz, N. G., Hellström, P., Angerbjörn, A., 2010. 
Strength of asymmetric competition between predators in food webs ruled by fluctuating prey: the case of foxes in tundra. Oikos 119 (1), 27-34.

Hendriks, A. J., Mulder, C., 2012. Delayed logistic and rosenzweig-macarthur models with allometric parameter setting estimate population cycles at lower trophic levels well. Ecological complexity 9, 43-54.

Hofbauer, J., Sigmund, K., 1998. Evolutionary Games and Population Dynamics. Cambridge University Press.

Holt, R. D., 1984. Spatial heterogeneity, indirect interactions, and the coexistence of prey species. The American Naturalist 124 (3), 377-406.

Hutson, V., Vickers, G., 1983. A criterion for permanent coexistence of species, with an application to a two-prey one-predator system. Mathematical Biosciences 63 (2), 253-269.

Jansen, V. A., 1995. Regulation of predator-prey systems through spatial interactions: a possible solution to the paradox of enrichment. Oikos, 384-390.

Jansen, V. A., 2001. The dynamics of two diffusively coupled predator-prey populations. Theoretical Population Biology 59 (2), 119-131.

Jansen, V. A., de Roos, A. M., et al., 2000. The role of space in reducing predator-prey cycles. The geometry of ecological interactions: simplifying spatial complexity, 183-201.

Jensen, C. X., Ginzburg, L. R., 2005. Paradoxes or theoretical failures? the jury is still out. Ecological Modelling 188 (1), 3-14.

Kang, Y., Sasmal, S. K., Messan, K., 2015. A two-patch prey-predator model with dispersal in predators driven by the strength of predation. arXiv preprint arXiv: 1505.03820 .

Kareiva, P., Mullen, A., Southwood, R., 1990. Population dynamics in spatially complex environments: theory and data [and discussion]. Philosophical Transactions of the Royal Society B: Biological Sciences 330 (1257), 175-190.

Klepac, P., Neubert, M. G., van den Driessche, P., 2007. Dispersal delays, predator-prey stability, and the paradox of enrichment. Theoretical Population Biology 71 (4), 436-444.

Kot, M., 2001. Elements of Mathematical Ecology. Cambridge University Press.

Levin, S. A., 1974. Dispersion and population interactions. The American Naturalist 108 (960), 207-228.

Levin, S. A., 1976. Population dynamic models in heterogeneous environments. Annual Review of Ecology and Systematics 7 (1), 287-310.

Levy, D., Harrington, H. A., Van Gorder, R. A., 2016. Role of seasonality on predator-prey-subsidy population dynamics. Journal of Theoretical Biology 396, 163-181.

MacDonald, N., MacDonald, N., 2008. Biological Delay Systems: Linear Stability Theory. Vol. 9. Cambridge University Press.

May, R. M., 1972. Limit cycles in predator-prey communities. Science 177 (4052), 900-902.

Murdoch, W., Nisbet, R., McCauley, E., DeRoos, A., Gurney, W., 1998. Plankton abundance and dynamics across nutrient levels: tests of hypotheses. Ecology 79 (4), 1339-1356.

Neubert, M. G., Klepac, P., Van den Driessche, P., 2002. Stabilizing dispersal delays in predator-prey metapopulation models. Theoretical Population Biology 61 (3), 339-347.

Nevai, A. L., Van Gorder, R. A., 2012. Effect of resource subsidies on predatorprey population dynamics: a mathematical model. Journal of Biological Dynamics 6 (2), 891-922.

Ott, E., 2002. Chaos in Dynamical Systems. Cambridge University Press.

Rosenzweig, M. L., MacArthur, R. H., 1963. Graphical representation and stability conditions of predator-prey interactions. The American Naturalist 97 (895), 209-223.

Rosenzweig, M. L., et al., 1971. Paradox of enrichment: destabilization of exploitation ecosystems in ecological time. Science 171 (3969), 385-387.

Roth, J. D., 2002. Temporal variability in arctic fox diet as reflected in stablecarbon isotopes; the importance of sea ice. Oecologia 133 (1), 70-77.

Roth, J. D., 2003. Variability in marine resources affects arctic fox population dynamics. Journal of Animal Ecology 72 (4), 668-676.

Scheffer, M., De Boer, R. J., 1995. Implications of spatial heterogeneity for the paradox of enrichment. Ecology 76 (7), 2270-2277.

Shen, L., Van Gorder, R. A., 2017. Predator-prey-subsidy population dynamics on stepping-stone domains. Journal of Theoretical Biology 420, 241-258.

Strogatz, S. H., 2014. Nonlinear Dynamics and Chaos: With Applications to Physics, Biology, Chemistry, and Engineering. Westview Press.

Turchin, P., 2003. Complex Population Dynamics: a Theoretical/Empirical Synthesis. Vol. 35. Princeton University Press.
Wall, E., Guichard, F., Humphries, A. R., 2013. Synchronization in ecological systems by weak dispersal coupling with time delay. Theoretical Ecology $6(4), 405-418$.

Weisser, W. W., Hassell, M. P., 1996. Animals' on the move'stabilize hostparasitoid systems. Proceedings of the Royal Society of London B: Biological Sciences 263 (1371), 749-754.

Weisser, W. W., Jansen, V. A., Hassell, M. P., 1997. The effects of a pool of dispersers on host-parasitoid systems. Journal of Theoretical Biology 189 (4), 413-425.

Winston, E., 1971. The global existence of solutions of delay differential equations. Journal of Differential Equations 10 (3), 392-402.

Wolf, A., Swift, J. B., Swinney, H. L., Vastano, J. A., 1985. Determining lyapunov exponents from a time series. Physica D: Nonlinear Phenomena 16 (3), 285-317.

Wrigley, R. E., Hatch, D. R., 1976. Arctic fox migrations in manitoba. Arctic, $147-158$.

Yang, Z., Liu, X., Zhou, M., Ai, D., Wang, G., Wang, Y., Chu, C., Lundholm, J. T., 2015. The effect of environmental heterogeneity on species richness depends on community position along the environmental gradient. Scientific reports $5,15723$.

Zhang, H., Huang, T., Dai, L., 2015a. Nonlinear dynamic analysis and characteristics diagnosis of seasonally perturbed predator-prey systems. Communications in Nonlinear Science and Numerical Simulation 22 (1), 407-419.

Zhang, Y., Lutscher, F., Guichard, F., 2015b. The effect of predator avoidance and travel time delay on the stability of predator-prey metacommunities. Theoretical Ecology 8 (3), 273-283. 